\title{
Portfolio Selection Under Uncertainty: a new methodology for computing relative-robust solutions
}

\author{
Sandra Caçador ${ }^{\mathrm{a}, \mathrm{b}, \mathrm{c}, 1}$, Joana Matos Dias ${ }^{\mathrm{b}, \mathrm{c}, \mathrm{d}}$ and Pedro Godinho ${ }^{\mathrm{b}, \mathrm{c}}$ \\ ${ }^{a}$ Higher Institute for Accountancy and Administration of the University of Aveiro, R. Associação \\ Humanitária dos Bombeiros Voluntários de Aveiro, Aveiro 3810-500, Portugal \\ ${ }^{b}$ Centre for Business and Economics Research (CeBER), Faculty of Economics of the University of \\ Coimbra, Av. Dias da Silva, 165, Coimbra 3004-512, Portugal \\ ${ }^{c}$ Faculty of Economics of the University of Coimbra, Av. Dias da Silva, 165, Coimbra 3004-512, \\ Portugal \\ ${ }^{d}$ Institute for Systems Engineering and Computers at Coimbra, Rua Antero de Quental, $N^{o} 199$, Coimbra \\ 3000 - 033, Portugal \\ E-mail: sandracacador@ua.pt [F. Author]; joana@fe.uc.pt [S. Author]; pgodinho@fe.uc.pt [T. Author]
}

\begin{abstract}
In this paper, a new methodology for computing relative-robust portfolios based on minimax regret is proposed. Regret is defined as the utility loss for the investor resulting from choosing a given portfolio instead of choosing the optimal portfolio of the realized scenario. The absolute robust strategy was also considered and, in this case, the minimum investor's expected utility in the worst-case scenario is maximized. Several subsamples are gathered from the in-sample data and for each subsample a minimax regret and a maximin solution are computed, to avoid the risk of overfitting. Robust portfolios are computed using a genetic algorithm, allowing the transformation of a 3-level optimization problem in a 2-level problem. Results show that the proposed relative-robust portfolio generally outperforms (other) relative-robust and non-robust portfolios, except for the global minimum variance portfolio. Furthermore, the relative-robust portfolio generally outperforms the absolute-robust portfolio, even considering higher risk aversion levels.
\end{abstract}

Keywords: Robust optimization, portfolio selection, relative robustness, minimax regret.

This is the peer reviewed version of the following article: Caçador, S., Dias, J.M. and Godinho, P. (2019), Portfolio selection under uncertainty: a new methodology for computing relative-robust solutions. Intl. Trans. in Op. Res. doi:10.1111/itor.12674, which has been published in final form at https://doi.org/10.1111/itor.12674. This article may be used for non-commercial purposes in accordance with Wiley Terms and Conditions for Use of Self-Archived Versions.

\footnotetext{
${ }^{1}$ Author to whom all correspondence should be addressed (e-mail: sandracacador@ua.pt).
} 


\section{Introduction}

Deciding how to optimally allocate the investor's wealth among all possible investment alternatives requires the consideration of the tradeoff between expected return and risk, and this is a difficult problem. Actually, the investor has to decide what to do today, without knowing what the future will bring regarding the different investment alternatives. In this context, the concept of optimal solution is not well defined. We can think of representing the inherent uncertainty by using a set of parameters that are, most of the times, calculated by observing past data. However, as it is not possible to foresee all potential realizations of the uncertain values, it is also not possible to assume that these parameters are a truthful representation of future uncertainty. A given portfolio can be optimal considering a given set of problem parameters and still present a poor performance as the uncertainty is resolved. If the uncertainty that arises from the impossibility to know the future values of the parameters at the decision-making moment is ignored, then the calculated optimal portfolio will be extremely sensitive to small variations in the problem parameters and may thus show a poor performance when applied to new data (Best and Grauer, 1991a, 1991b; Chopra and Ziemba, 1993; Jagannathan and Ma, 2003).

In this regard, some methodologies that explicitly incorporate uncertainty into the optimization model can be applied in order to mitigate the impact of the estimation errors in the classical meanvariance optimization problems. An example is the robust optimization methodology, whose roots can be found in the field of robust control theory.

Robust optimization has emerged as a computationally attractive alternative to other methodologies, like stochastic programming or dynamic programming, since it requires relatively general and simple assumptions about the probability distributions of the uncertain parameters (Fabozzi et al., 2007). The robust formulation of an optimization problem considers not only the nominal values of the uncertain parameters but also the deviations from these nominal values. Uncertainty in the parameters can be described by uncertainty sets that contain all possible values or only the most likely values of the uncertain parameters, with their sizes defining the level of uncertainty admitted or, equivalently, the desired level of robustness.

The first to apply this idea was Soyster (1973), who presented a linear optimization model to compute a solution that was feasible for all possible values of an uncertain parameter belonging to a convex set (Ben-Tal, El Ghaoui and Nemirovski, 2009). While Soyster's approach achieved the desired outcome of immunizing the optimal solution against parameter uncertainty, it was widely considered too conservative for practical implementation (Bertsimas and Thiele, 2006). More than twenty years later, other authors addressed the overconservatism within Soyster's approach and robust optimization began to establish itself as a methodology with applications in many fields of knowledge, including portfolio theory.

Two main approaches are considered in the robust portfolio optimization literature. The most prevalent is the absolute robust optimization approach where the optimal solution is computed assuming the worst possible realization within the uncertainty set for the uncertain parameters. Since assuming that the worst scenario will happen might result in too conservative decisions, one could analyze robustness in a relative manner. In the relative robust optimization approach the objective is to guarantee that the maximum difference between the optimal objective function value for each scenario (considering the optimal solution for that scenario) and the objective function value obtained for the same scenario by the robust solution (that is not scenario dependent) is minimized.

In this paper, a new methodology for computing relative-robust portfolios is presented, based on the optimization of the investor's expected utility. Since empirical applications of the relative robust models are lacking within the portfolio optimization theory, an analysis of the real benefits of the relative robust methodology is performed. For that purpose, an empirical application is conducted in order to assess and compare the performance of the proposed relative-robust portfolio to (other) relative-robust and non-robust portfolios already described in the portfolio theory literature. A comparison of the performance of relative-robust and absolute-robust 
portfolios is also presented and conclusions are drawn regarding the real benefits of the proposed methodology from the investors' perspective.

This paper contributes for this field of research in several ways. First, a new methodology for computing relative-robust portfolios based on minimax regret is presented. Second, a 3-level optimization problem is transformed into a 2-level problem by using an evolutionary algorithm to solve the relative-robust portfolio optimization problem and find the relative-robust solution. Third, the real benefits of applying the relative robustness approach in portfolio selection are analyzed by comparing in-sample and out-of-sample performances of relative-robust and nonrobust portfolios, which highlight the main strengths of the proposed methodology. Fourth, the effect of considering long-term past data over short-term past data in the definition of the uncertainty set is investigated by using in-sample sets of different lengths in the empirical analysis. Finally, the relevance of the proposed robust models is analysed for different levels of risk aversion and the performances of relative robust and absolute robust approaches are compared.

For the empirical analysis, historical daily data from January 1992 to December 2016 (25 years) of the stocks of the DAX index were collected from Thompson Reuters Datastream. Different methodological approaches are implemented and the corresponding optimal portfolios are computed, in particular the proposed relative-robust (RR) portfolio, the absolute-robust (AR) portfolio, the classical Mean-Variance (MV) portfolio, the Global Minimum Variance (GMV) portfolio, the Equally Weighted (EW) portfolio and the relative-robust (WS) portfolio presented by Xidonas et al. (2017). The latter is, to the best of the authors' knowledge, the only known contribution with empirical applications within the relative-robust portfolio optimization approach. The performances of the different computed portfolios are compared considering both in-sample and out-of-sample data, for return, risk, modified Sharpe ratio and regret.

The results of this study suggest that reducing the in-sample period length increases the exposure of the computed portfolios to individual stocks while it seems to improve the overall out-ofsample performance of the AR, the RR and the GMV portfolios and substantially deteriorates the out-of-sample performance of the MV portfolio. Regardless of the in-sample period length, it can be observed that the RR portfolio is highly diversified, assigning non-zero weights to the majority of the assets.

The overall results support previous findings concerning the sensitivity of the MV portfolio to the estimation error and the effects of the input uncertainty in the optimization process (Best and Grauer, 1991b; Chopra and Ziemba, 1993; Jagannathan and Ma, 2003; DeMiguel, Garlappi and Uppal, 2009), as well as the outperformance of the GMV portfolio (Chan, Karceski and Lakonishok, 1999; Jagannathan and Ma, 2003).

Furthermore, the results of this study suggest that the proposed relative robust model generates optimal portfolios that consistently present low risk, and (non-negative) attractive returns, standing out as one of the very few optimal portfolios with no poor performances. In fact, the RR portfolio outperforms the MV portfolio and the EW portfolio in many of the windows under analysis, even outperforming the GMV portfolio in some windows, which enhance the relevance of the proposed methodology among non-robust portfolio optimization methodologies. When compared to the minimax regret model presented by Xidonas et al. (2017), the proposed relative robust approach seems to provide more consistent results concerning the out-of-sample performance of the generated portfolios.

Finally, the comparison of the proposed relative robust and absolute robust models leads to important conclusions concerning the real benefits of the proposed methodology from the investors' perspective. The findings suggest that the proposed RR portfolio generally outperforms the proposed AR portfolio, even when higher levels of risk aversion are considered.

The remainder of the paper proceeds as follows. A brief literature review is presented in Section 2. In Section 3, the proposed model is presented and the process for computing the relative-robust solution is described. The results of the empirical analysis are presented in Section 4. Finally, in Section 5, main conclusions and suggestions for future research are highlighted. 


\section{Literature review}

\subsection{Portfolio selection problem}

The formulation of the decision-making problem concerning the optimal allocation of an investor's wealth among the possible investment choices was formally presented, for the first time, by Harry Markowitz $(1952,1959)$. He established two essential concepts that became the basis of the modern portfolio theory. The first one was the joint quantitative assessment of return and risk by considering the expected return and covariance of the securities, which laid the foundations for the diversification principle. The second one was the formulation of the portfolio selection problem as an optimization problem: the selection of the securities must guarantee the maximization of the expected return of the portfolio while holding constant the risk of the portfolio or, equivalently, guarantee the minimization of the portfolio risk while holding constant its expected return.

As pointed out by Constantinides and Malliaris (1995), the description of asset returns in terms of a probability distribution made by Fisher (1906), the definition of investment preferences as indifference curves in the mean-variance space presented by Marschak (1938), the axiomatic theory of choice under uncertainty developed by Von Neumann and Morgenstern (1947), and the risk measurement framework presented by Bernoulli (1954), were decisive contributions for the development of Markowitz's portfolio selection theory.

Markowitz supported his mean-variance analysis of the investment decision problem on meanvariance approximations to the investor's expected utility, which he described as the "MVapproximate" approach: after generating the mean-variance efficient frontier, one must choose the efficient portfolio that maximizes the mean-variance function which approximates the expected value of the investor's utility (Markowitz, 2014). Although the empirical support of the "MV-approximate" approach, presented by Markowitz (1959), assumed a quadratic utility function and expected returns and covariance matrix of returns as estimators of the input parameters, neither normal probability distribution of asset returns nor quadratic utility functions are necessary conditions for the applicability of modern portfolio theory. In fact Levy and Markowitz (1979) proved that expected utility can be approximated by a function of mean and variance for various utility functions and historical distributions of returns.

While the investment decision problem can be generalized into a much wider class of problems (the expected utility maximization problems), Markowitz's mean-variance framework presented itself as a simpler approach and served as a starting point for the numerous contributions that appeared in the following decades. These contributions involved, but were not limited to, the following research areas: return-generating functions, index models and asset pricing models (Sharpe, 1964; Ross, 1976; Ingersoll, 1987; Elton and Gruber, 1992; Fama and French, 1992; Roll and Ross, 1994; Fama, 1996), risk measures (Hendricks, 1997; Artzner et al., 1999; Rockafellar and Uryasev, 2000; Szegö, 2005), incorporating constraints in the portfolio selection model (Clarke, De Silva and Thorley, 2002; Jagannathan and Ma, 2003; Scherer and Xu, 2007) and multi-criteria portfolio optimization (Zopounidis and Doumpos, 2002; Doerner et al., 2004; Steuer, Qi and Hirschberger, 2008; Deb, 2011).

With the development of computer tools, the computational complexity of the expected utility maximization problems is no longer an inconvenience and the use of this wider class of problems becomes more frequent in the portfolio theory literature (Jean, 1971; de Athayde and Flôres, 2004; Harvey et al., 2010; Biagini and Pinar, 2017).

Following the maximization of the investor's utility approach, the portfolio selection problem can be formulated as the maximization of the investor's wealth utility function. A wealth utility function shows how much utility (or how much happiness) a person derives from different levels of wealth or, equivalently, from different levels of return. Since a person's wealth is a function of the rate of return measuring the rate at which wealth is accumulated, the investor's utility of wealth and utility of return functions yield identical preference orderings (Francis and Kim, 2013). General assumptions are frequently used concerning investor's preferences, namely that an investor always prefers more to less utility, but that marginal utility decreases with increasing wealth (Fabozzi et al., 2007). The marginal utility of wealth is defined as the additional utility a 
person gets from a small change in her wealth. Thus, the marginal utility of wealth of every rational investor will always be positive. Additionally, rational investors are assumed to be riskaverse since, when faced with choosing between two investments with the same expected return but two different levels of risk, they prefer the one with the lower risk. Thus, positive but diminishing marginal utility is one of the characteristics economists expect to find in a realistic economic model (Francis and Kim, 2013).

Further assumptions for describing the behaviour of rational risk-averse investors include decreasing absolute risk aversion (DARA) and constant relative risk aversion (CRRA): "Although there is much less consensus regarding relative risk aversion than absolute risk aversion, it is often assumed that constant relative risk aversion is consistent with most investors' behaviour (...)" while " $(\ldots)$ there is general consensus that most investors exhibit decreasing absolute risk aversion" (Francis and Kim, 2013). The absolute risk aversion is a measure of how the investor's preference for risk changes with a change in wealth. If the investor wants to put more wealth in risky investments as his or her wealth increases, it is said that the investor exhibits DARA. So, as the investor gets richer, he or she becomes less risk averse. The relative risk aversion is a measure of how the percentage of wealth invested in risky assets changes with a change in wealth. An investor exhibiting CRRA maintains the same percentage in the risky investments when his or her wealth changes.

\subsection{Robust portfolio optimization}

The classical portfolio selection problem, as presented by Markowitz, completely disregards the uncertainty of the expected asset's returns and the covariance matrix of assets' returns. It is assumed that these parameters are capable of representing the inherent uncertainty associated with the investment returns. Actually, as these parameters are, most of the times, calculated from past data, they are themselves subject to uncertainty. Not acknowledging the uncertainty in the models' parameters substantially degrades the performance of the optimal solution calculated using these models (Best and Grauer, 1991a, 1991b; Chopra and Ziemba, 1993).

As previously mentioned, the robust optimization methodology allows the incorporation of uncertainty into the optimization model, mitigating the impact of the estimation errors in the portfolio selection problem. According to Hauser, Krishnamurthy, and Tütüncü (2013, p. 1), the robust optimization methodology aims to "(...) mitigate the effects of uncertainty and obtain a solution that is guaranteed to perform reasonably well for all, or at least most, possible realizations of the uncertain input parameters".

One may be interested in guaranteeing that the solution will perform reasonably well relatively to its feasibility, or its optimality, or both its feasibility and its optimality. Therefore, different concepts of robust solution emerged in the literature (Gabrel, Murat and Thiele, 2014). When the uncertainty affects the feasibility of a solution, which happens when the uncertain parameter is included in the constraints of the optimization model, the robust optimization methodology aims to ensure that the solution will be feasible for all, or at least most, realizations of the uncertain parameters. When the uncertain parameter is included in the objective function of the optimization model, affecting the optimality of a solution, the robust optimization methodology aims to ensure that the feasible solution achieves exactly or approximately the optimum value of the objective function, regardless of the realizations of the uncertain parameters.

Most of the studies in the robust portfolio optimization field seek to optimize the worst-case realization of the objective function, where the worst-case is computed over the uncertainty set. This constitutes the absolute robust optimization approach (Hauser, Krishnamurthy and Tütüncü, 2013). Initial contributions, under the absolute robust design, focused on the formulation of the robust counterparts of classic portfolio optimization problems or the development of deterministic algorithms in order to solve them (El Ghaoui and Lebret, 1997; Ben-Tal and Nemirovski, 1998; Goldfarb and Iyengar, 2003; Halldórsson and Tütüncü, 2003). More recent contributions explored the close relationship between the structure of the uncertainty set and the risk measure selected (Natarajan, Pachamanova and Sim, 2009; Ben-Tal, Bertsimas and Brown, 2010; Paç and Pınar, 2014), analyzed the effects of the uncertainty sets' structure and scale (Lu, 2006, 2011; Roy, 2010; Gregory, Darby-Dowman and Mitra, 2011; Kalä1, Lamboray and Vanderpooten, 2012; Fabretti, 
Herzel and Pinar, 2014), and compared the characteristics of absolute-robust portfolios to classic portfolios (Kim et al., 2013; Kim, Kim and Fabozzi, 2013; Kim et al., 2014b).

Concerning the characteristics of absolute-robust portfolios, Kim, Kim and Fabozzi (2013) presented a robust approach for the maximization of the risk-adjusted expected return problem, in which the uncertainty was admitted only in the expected assets' return. Based on a factor model for defining the vector of asset returns and considering box and ellipsoidal uncertainty sets, the authors analyzed the characteristics of their robust portfolio and classical mean-variance and global minimum-variance portfolios, computed assuming short-sales constraints and specific values for the risk-averse coefficient. In particular, they analyzed the level of diversification of portfolios by comparing the number of assets with non-zero weights contained in the portfolios, the exposure of the portfolios to individual stocks by comparing the absolute value of the assets' weight, the volatility of the portfolios in relation to the market portfolio by comparing portfolio betas, and the correlation between weight and beta of the assets composing the portfolio. Their results revealed that the robust portfolio is less diversified than the non-robust portfolio since it contains fewer stocks with non-zero weights, shows lower exposure to individual stocks since it reveals assets' weights with lower maximum absolute values, it is more exposed to the systematic risk since it has higher portfolio beta and it shows low correlation between weight and beta of the stocks composing the portfolio. The authors have also found that the robust solution based on box uncertainty set has a lower beta and fewer assets with non-zero weights than the robust portfolio based on ellipsoidal uncertainty set, revealing itself as a portfolio less sensitive to systematic risk but less diversified and, thus, more exposed to the individual risk of each asset. On the other hand, the robust portfolio based on an ellipsoidal uncertainty set has higher positive portfolio beta and stronger negative correlation between weight and beta of the stocks composing the portfolio. Based on the same optimization framework, Kim et al. (2013) showed that, comparatively to the mean-variance portfolio, absolute-robust portfolios consistently show stronger correlation with the three fundamental factors used in the Fama-French factor model ${ }^{2}$ and that the intensity of the correlation increases as the robustness of the portfolio is increased ${ }^{3}$. Furthermore, the authors found that the absolute-robust portfolio based on ellipsoidal uncertainty set revealed stronger correlation with the three fundamental Fama-French factors comparatively to the robust portfolio based on box uncertainty set.

The absolute robust approach might not be adequate for all investors. One example is when decision makers' performances are judged relative to their peers' performance. They might prefer to make decisions that avoid falling severely behind their competitors under all scenarios, rather than protecting themselves against the worst-case scenarios. Following this idea, Kouvelis and $\mathrm{Yu}$ (1997) explored the concept of relative robustness by analyzing the worst-case in a relative manner, considering the best possible solution under each scenario.

The notion of relative robustness can be explained resorting to a generic portfolio optimization model which includes the uncertain parameters in the objective function (Cornuejols and Tütüncü, 2006). Let $x \in \mathbb{R}^{N}$ be the weight combination vector defining the investor's portfolio, $p$ the vector defining the input parameters and $X$ the set of feasible solutions. Then it is possible to define the following optimization model:

$\max _{x \in \mathbb{R}^{N}} f(x, p)$ s.t. $x \in X$

where $f$ represents a generic objective function that depends on $x$ and $p$.

For a given $p$, let $z^{*}(p)$ and $x^{*}(p)$ denote, respectively, the optimal objective function value and the vector of optimal decision variables values of problem (1). If $x$ is chosen as the decision vector

\footnotetext{
${ }^{2}$ The three Fama-French factors are: the market risk premium corresponding to the difference between the expected return of the market portfolio and the risk-free rate; the small minus big (SMB) corresponding to the difference between the return on a portfolio of small stocks and the return on a portfolio of large stocks; and the high minus low (HML) corresponding to the difference between the return on a portfolio of high-book-to-market stocks and the return on a portfolio of low-book-to-market stocks (Fama and French, 1996).

${ }^{3}$ The level of robustness was adjusted through the value of the parameter that defines the scale of the uncertainty set, i.e. that allows expanding or reducing the uncertainty set.
} 
when $p$ is the vector of realized parameter values, then the regret associated with having chosen $x$ rather than $x^{*}(p)$ is defined as follows:

$R_{p}(x):=z^{*}(p)-f(x, p)=f\left(x^{*}(p), p\right)-f(x, p)$.

Since regret cannot be measured before the realization of vector $p$, it is possible to consider the maximum regret function instead, which provides an upper bound on the true regret:

$R(x):=\max _{p \in U} R_{p}(x):=\max _{p \in U}\left(z^{*}(p)-f(x, p)\right)$

where $U$ represents the uncertainty set, i.e. the set of possible scenarios/realizations for the vector of realized parameters $p$.

Thus, a relative-robust solution $x$ corresponds to the weight combination vector that minimizes the maximum regret function and therefore solves the relative robust optimization model:

$\min _{x \in X} \max _{p \in U}\left(z^{*}(p)-f(x, p)\right)$

Hence, the relative robust optimization approach leads to three-level optimization problems, in contrast with the absolute robust optimization framework which leads to two-level optimization problems (Cornuejols and Tütüncü, 2006; Hauser, Krishnamurthy and Tütüncü, 2013).

Kouvelis and $\mathrm{Yu}$ (1997, p. xi) have described a collection of discrete optimization problems based on the relative robustness framework. This framework considers a minimax regret criterion, and can be applied to models with discrete decision variables that allow the use of convex and combinatorial optimization techniques. There are also works considering continuous portfolio optimization problems, as the work of Hauser, Krishnamurthy, and Tütüncü (2013), that assume ellipsoidal uncertainty. The cited works show that it is possible to reduce the relative robust formulation resulting from many optimization problems to one or a series of single-level deterministic optimization problems that can be solved using deterministic algorithms. These observations can drive the development of specialized algorithms for relative robust optimization applications.

The only contribution with empirical applications considering the relative-robust portfolio optimization approach that the authors are aware of was developed by Xidonas et al. (2017). Considering a two-objective optimization problem, and seeking to minimize the mean absolute deviation $\left(f_{1}\right)$ and to maximize the expected portfolio return $\left(f_{2}\right)$, the authors applied the weighting method in order to calculate the Pareto optimal solution. Assuming that there are $g$ weight combinations, each one characterized by the weight vector $\left(w_{1}^{g}, w_{2}^{g}\right)$, with $w_{1}^{g}$ and $w_{2}^{g}$ representing the weights of the objective functions $f_{1}$ and $f_{2}$, respectively, and $w_{1}^{g}+w_{2}^{g}=1, \forall g$. The optimization model corresponding to the maximization of the weighted sum of the two individual objective functions $f_{1}$ and $f_{2}$, is given by

$$
\begin{aligned}
& \operatorname{MMR}(g)=\min y_{g} \\
& \text { s.t. } w_{1}^{g} \frac{f_{1, \max }^{c}-f_{1}^{c}(x)}{f_{1, \text { max }}^{c}-f_{1, \min }^{c}}+w_{2}^{g} \frac{f_{2}^{c}(x)-f_{2, \min }^{c}}{f_{2, \text { max }}^{c}-f_{2, \text { min }}^{c}} \geq\left(1-y_{g}\right) z_{c}^{g}, \quad c \in C, \\
& \quad x \in F
\end{aligned}
$$

where $f_{1, \text { max }}^{c}, f_{1, \text { min }}^{c}, f_{2, \text { max }}^{c}$ and $f_{2, \text { min }}^{c}$ are the maximum and the minimum values of the objective functions $f_{1}$ and $f_{2}$, respectively, for a given scenario $c \in C$, and $z_{c}^{g}$ is the optimal value of the weighted sum of the objective functions for scenario $c$ and weight combination $g$. The empirical test performed by Xidonas et al. (2017) considered eleven different weight combinations for the discretization of the weight space and an uncertainty set with 5 scenarios of return and risk evolution. The results show that the minimax regret portfolio includes more stocks than the optimal portfolios of the individual scenarios, in all the weight combinations, representing a more disperse allocation of the total investment universe. Furthermore, the in-sample performance 
analysis revealed that the area of the Pareto front that corresponds to minimizing risk against maximizing return (i.e. when minimizing risk is weighted more than maximizing return) provides more robust solutions in terms of the minimax regret criterion, thus lower minimax regret values, where the minimax regret expresses how far one is from the individual optima of each scenario in the worst-case. No out-of-sample performance analysis was presented in this study. For further details see Xidonas et al. (2017).

\section{Computing relative-robust and absolute-robust solutions}

\subsection{The robust optimization models}

Let $W$ represent the wealth invested in a set of $N$ stocks, $x \in \mathbb{R}^{N}$ be the weight combination vector defining the investor's portfolio, $r_{t+1}^{p}$ be the portfolio return at time period $t+1$ and $u($.$) be the$ investor's utility function. The expected utility portfolio maximization problem can be defined as

$\max E\left[u\left(W\left(1+r_{t+1}^{p}\right)\right)\right]$

s.t. $x \in X$

where $X$ represents the set of feasible solutions. Since utility preference orderings are invariant under a positive linear transformation of the utility function and as pointed out in section 2.1, maximizing the expected utility of the wealth function yields identical preference orderings as maximizing the expected utility of the rate of return at which wealth is accumulated.

For describing the investor's preferences, consider the power utility function of the investor with constant relative risk aversion (CRRA) preferences over wealth and constant relative risk aversion parameter $\left(\gamma \in \mathbb{R}^{+} \backslash\{1\}\right)$, defined as:

$u\left(1+r_{t+1}^{p}\right)=\frac{\left(1+r_{t+1}^{p}\right)^{1-\gamma}}{1-\gamma}$

As an approximation to the expected utility of the portfolio, $E\left[u\left(1+r_{t+1}^{p}\right)\right]$, consider the second order Taylor expansion of the expected utility function around the expected return of the portfolio, $E\left[r_{t+1}^{p}\right]$, given by:

$E\left[u\left(1+r_{t+1}^{p}\right)\right]=\frac{\left(1+E\left[r_{t+1}^{p}\right]\right)^{1-\gamma}}{1-\gamma}-\frac{\gamma}{2}\left(1+E\left[r_{t+1}^{p}\right]\right)^{-\gamma-1} V\left[r_{t+1}^{p}\right]$

where $V\left[r_{t+1}^{p}\right]$ is the portfolio expected variance. This study does not extend to moments higher than the second moment (variance), as the applications of the expected utility maximization problems usually do, since previous works have shown that the logarithmic and power utility functions are fairly insensitive to higher moments (Cremers, Kritzman and Page, 2003, 2005).

For the empirical application, CRRA preferences and the utility power function previously presented were assumed. Uncertainty was considered both in the vector of assets' returns and in the covariance matrix of returns. A joint uncertainty set was defined for both uncertain parameters as in Kim et al. (2014a).

Consider a universe of $N$ assets for which $P$ observations regarding consecutive trading days are known and let $r_{j n}$ be the return of asset $n$ at time $j$. Each scenario, represented by $s$, will be built considering the observations associated with different sampling periods of length $J$. Let $z(s)$ represent a random value such that $z(s) \in\{1, \ldots, P-J+1\}$. All the observations between $z(s)$, which defines the first time period, and $z(s)+J-1$, which defines the last time period, will be used for building scenario $s$. The sample returns of the $N$ assets during this randomly generated time window of length $J$ will be represented by matrix $M^{s} \in \mathbb{R}^{J \times N}$. The vector with the returns of the different assets at time $j$ will be represented by $r_{j}^{S}$. Let $\mu^{S}$ and $\Sigma^{S}$ be the sample mean vector and the sample covariance matrix for the sample set used for building the scenario $s$, respectively. Then, the joint uncertainty set $U$, corresponding to a finite set of scenarios, is defined by 
$U=\left\{s_{1}, s_{2}, \ldots, s_{S}\right\}$

where each $s_{i}$ is defined in the following way (to avoid cluttering the notation, we drop the index $i$ from $s_{i}$ in this definition):

$s=\left(\mu^{s}, \Sigma^{s}\right)$,

$\mu^{s}=\left[\begin{array}{c}\frac{1}{J} \sum_{j=z(s)}^{Z(s)+J-1} r_{j 1} \\ \vdots \\ \frac{1}{J} \sum_{j=Z(s)}^{Z(s)+1-1} r_{j N}\end{array}\right]$

and

$\Sigma^{s}=\frac{1}{J-1} \sum_{j=z(s)}^{z(s)+J-1}\left(r_{j}^{S}-\mu^{s}\right)\left(r_{j}^{S}-\mu^{s}\right)^{T}$

The proposed relative robust minimax regret approach defines regret as the utility loss for the investor resulting from choosing a portfolio characterized by the weight combination vector $x$ instead of choosing $x^{s *}$ which corresponds to the optimal solution under scenario $s$.

Let $r_{t+1}^{p *}$ be the realized return of the optimal portfolio $x^{s *}$ given by $r_{t+1}^{p *}=\sum_{n=1}^{N} x_{n}^{*} r_{t+1, n}$, where $x_{n}^{*}$ represents the optimal weight of asset $n$. Equivalently, $r_{t+1}^{p}(x)$ corresponds to the expected return of the portfolio characterized by the weight combination vector $x$, which is given by $r_{t+1}^{p}(x)=\sum_{n=1}^{N} x_{n} r_{t+1, n}$, where $x_{n}$ represents the weight of asset $n$. The regret associated to scenario $s, R^{S}$, is defined by

$R^{s}(x):=E\left[u^{s}\left(1+r_{t+1}^{p *}\right)\right]-E\left[u^{s}\left(1+r_{t+1}^{p}(x)\right)\right]$

and the maximum regret function, $R(x)$, is defined by

$R(x):=\max _{s \in U} E\left[u^{s}\left(1+r_{t+1}^{p *}\right)\right]-E\left[u^{s}\left(1+r_{t+1}^{p}(x)\right)\right]$.

The minimax regret solution $x$ corresponds to the weight combination vector that solves the relative robust optimization model:

$\min _{x \in X} \max _{s \in U} E\left[u^{s}\left(1+r_{t+1}^{p *}\right)\right]-E\left[u^{s}\left(1+r_{t+1}^{p}(x)\right)\right]$,

where the set of feasible solutions is defined as $X=\left\{x \in \mathbb{R}^{N}: \sum_{n=1}^{N} x_{n}=1 \wedge x_{n} \geq 0, \forall n=\right.$ $1, \ldots, N\}$.

Hence, assuming CRRA preferences, the optimization problem (12) can be defined by

$\min _{x \in X} \max _{s \in U}\left[\left(\frac{\left(1+x^{S * T} \mu^{s}\right)^{1-\gamma}}{1-\gamma}-\frac{\gamma}{2}\left(1+x^{S * T} \mu^{S}\right)^{-\gamma-1} x^{S * T} \Sigma^{S} x^{S *}\right)-\left(\frac{\left(1+x^{T} \mu^{s}\right)^{1-\gamma}}{1-\gamma}-\frac{\gamma}{2}(1+\right.\right.$

$\left.\left.\left.x^{T} \mu^{S}\right)^{-\gamma-1} x^{T} \Sigma^{S} x\right)\right]$

Note that problem (13) is a 3-level optimization problem. The computation of $x^{s *}$, which is the optimal portfolio for scenario $s$, constitutes the first optimization level. The inner maximization problem in (13) constitutes the second optimization level and allows the computation of the maximum regret for each $s \in U$, providing an upper bound on the true utility loss for the investor. 
Finally, the third optimization level corresponds to the outer minimization problem in (13), which gives the optimal solution that minimizes the maximum regret for all $s \in U$.

For the absolute-robust portfolio optimization approach, the absolute-robust portfolio (maximin solution) corresponds to the weight combination vector that solves the absolute robust optimization model defined by:

$\max _{x \in X} \min _{s \in U} E\left[u^{s}\left(1+r_{t+1}^{p}(x)\right)\right]$.

\subsection{Computing the robust portfolios}

First, the computation of the relative-robust portfolio is explained. To avoid reaching a solution strongly dependent on the underlying in-sample data, two disjoint subsamples are randomly gathered from the in-sample data: an estimation subsample used to estimate the inputs of the model and a validation subsample used to evaluate its performance.

From the estimation subsample, an uncertainty set $U$ is constructed by calculating the $S$ scenarios. For that purpose, an estimation window is randomly selected from the estimation subsample and the sample mean and the sample covariance matrix are computed. Then, for each scenario $s \in U$ the following problem is solved

$\max _{x \in X}\left(\frac{\left(1+x^{T} \mu^{s}\right)^{1-\gamma}}{1-\gamma}-\frac{\gamma}{2}\left(1+x^{T} \mu^{S}\right)^{-\gamma-1} x^{T} \Sigma^{S} x\right)$,

in order to determine the optimal solution $x^{s *}$, which represents the portfolio on the Markowitz's efficient frontier that maximizes the investor's expected utility. This constitutes the first optimization process of the proposed 3-level optimization problem and was performed using CPLEX solver.

After computing the optimal solutions for each scenario $s \in U$, the relative robust optimization problem (13) is solved using the Genetic Algorithm (GA) toolbox from Matlab R2018a. For this purpose, a fitness function that maximizes the regret as presented in (11) and corresponding to the utility loss for the investor resulting from choosing a portfolio characterized by the weight combination vector $x$ instead of choosing the optimal solution for the estimation subsample period, was defined. An initial population composed of feasible solutions randomly generated was used. Further GA options used in this application include uniform mutation (with probability rate 0.15 ), tournament selection and a tolerance of $1 \mathrm{e}-16$ for the average relative change in the best fitness function value.

There are several deterministic methods available in the literature for solving similar minimax optimization problems (Yaman, Karasan and Pinar, 2007; Pınar and Burak Paç, 2014; Pınar, 2016; Paç and Pınar, 2018). In this work, a different path was taken. The proposed models are solved by a GA. Evolutionary algorithms, such as GA, allow researchers to solve more complex and combinatorial problems in a reasonable time (Soleimani, Golmakani and Salimi, 2009). Their application to optimization problems with non-linear or non-convex objective functions, in order to reduce the computational effort of finding the (near) optimal solution, is increasing in the portfolio theory literature (Streichert, Ulmer and Zell, 2004; Chang, Yang and Yhang, 2009; Soleimani, Golmakani and Salimi, 2009; Zhu et al., 2011; Kalayci et al., 2017). In this study, the use of GA allowed the second and third optimization levels to be solved simultaneously, reducing the computational effort to find the robust solutions. Furthermore, the efficiency of the GA as a tool to overcome the difficulties raised by computational complexity when solving (portfolio) optimization problems that address future uncertainty has also been reported by some authors (Jin and Branke, 2005; Yang, 2006). Gen and Cheng (2000) state that the multiple directional and global search performed by the GA where a population of potential solutions is maintained from population to population, is useful when exploring Pareto solutions, which is relevant for the investment decision problem since it is, in nature, a multi-objective optimization problem with 
(multiple) conflicting objectives. According to Shoaf and Foster (1998, p. 358), the “(...) GA can simultaneously minimize risk and maximize expected return (...) This flexibility allows the GA to discover portfolio opportunities which the more traditional [programming] approach misses". Finally, by applying both deterministic (when computing the optimal solution for each scenario) and evolutionary algorithms we are conducting a more efficient implementation of an algorithm capable of solving our problem, as highlighted by Gen and Cheng (2000, p. 107): "Because GAs, as a kind of meta-heuristics, provide us with great flexibility to incorporate conventional methods into the main framework, we can exploit the advantages of both GAs and conventional methods to establish much more efficient implementations to problems".

The random generation of both estimation and validation subsamples and the GA optimization is repeated several times, producing sets of different minimax regret solutions and different validation subsamples. Although the estimation and validation subsamples are gathered at the same time, the validation subsamples are used later in order to assess each one of the minimax regret solutions computed using the GA. By gathering both simultaneously, and defining them as disjoint sets, we guarantee that each minimax regret solution is tested at least in one validation subsample that does not overlap with its estimation subsample.

For testing each minimax regret solution in each one of the validation subsamples, regret was defined as the difference between the utility of the optimal portfolio (solution on the Markowitz's efficient frontier that maximizes the investor's expected utility) in the validation subsample period and the utility of the minimax regret solution. Then, the corresponding maximum regret is found. By considering the worst-case for each minimax regret solution, all possible bias, deriving from testing these solutions in validation subsamples that could overlap with their corresponding estimation subsamples, is avoided. Finally, the relative-robust portfolio defined as the minimax regret solution presenting the best performance in the worst-case is identified.

Figure 1 illustrates the flowchart of the proposed methodology for computing the relative-robust portfolio.

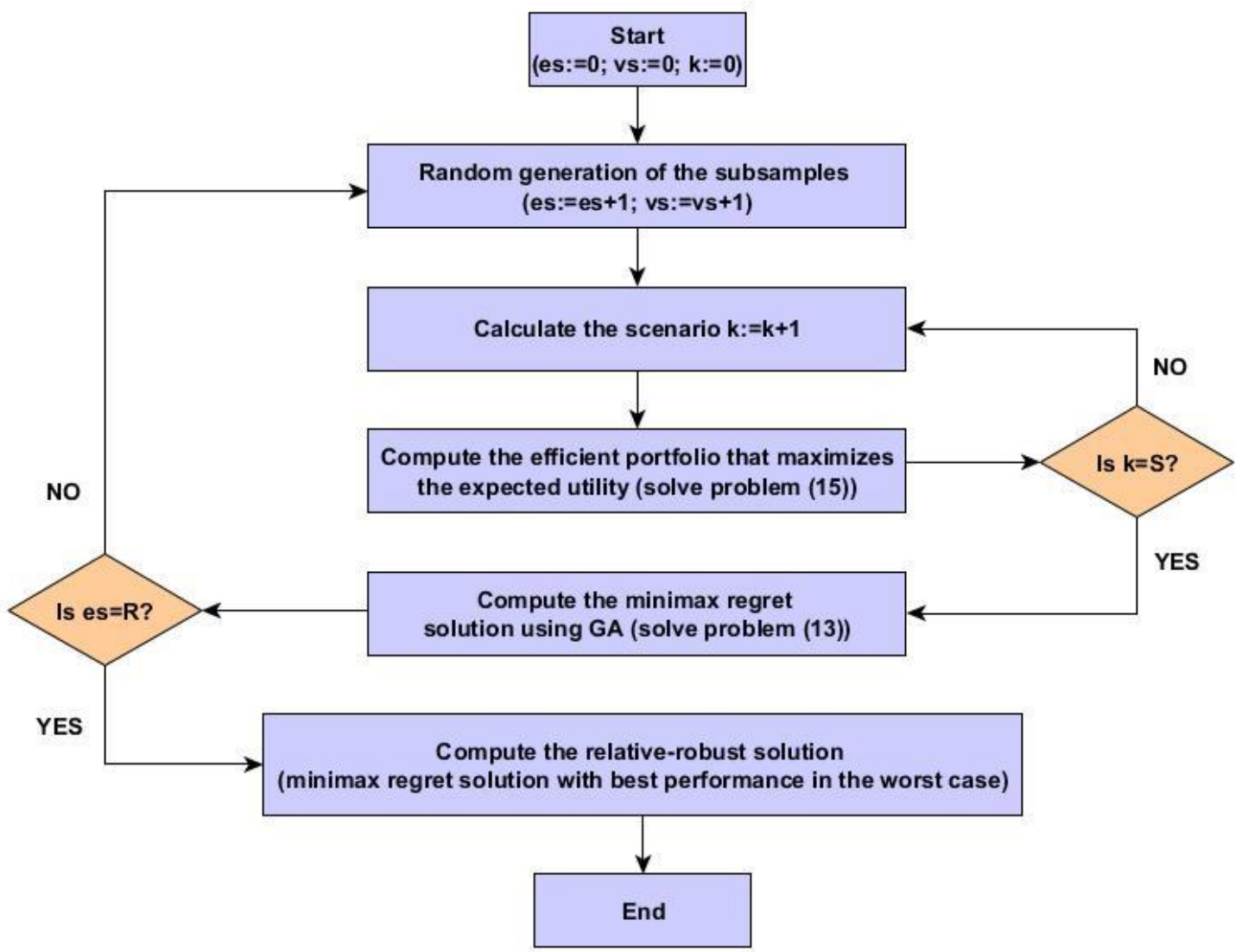


Figure 1: The flowchart of the method for computing the relative-robust solution. At the beginning of the process, the number of estimation subsamples (es), the number of validating subsamples (vs) and the number of scenarios $(k)$ are set to zero.

Concerning the computation of the absolute-robust portfolio, a similar process was applied. From each estimation subsample, an uncertainty set $U$ is constructed as previously described. After computing the $S$ scenarios, the maximin solution is calculated by solving problem (14) using the GA. In this case, the fitness function was defined as the minimum portfolio's expected utility for all the scenarios considered in $U$ (inner maximization problem in (14)). Hence, the optimization is performed assuming the worst-case performance over the whole uncertainty set. The initial population was composed by feasible randomly generated solutions. The GA options used were the same as the ones defined in the relative robust approach. Then, each maximin solution is tested in each one of the validation subsamples. For that purpose, the expected utility of the maximin solution in each of the validation subsample is calculated and the minimum expected utility is considered. Finally, the absolute-robust portfolio defined as the maximin solution presenting the best performance in the worst-case (highest minimum expected utility) is identified.

\section{Empirical analysis}

\subsection{Data and parameter settings}

For the empirical analysis, historical daily data from January 1992 to December 2016 (25 years) of the stocks of the DAX index was collected from Thompson Reuters Datastream.

The empirical strategy was performed using rolling windows of two different lengths. The first approach considered a rolling window with a constant length of 16-years: 15-years data to perform in-sample estimations and an out-of-sample evaluation period of 1-year. Thus, for this approach, the first window ranges from January 1992 to December 2007 (in-sample period from 1992 to 2006 and out-of-sample consisting of 2007) while the last window ranges from January 2001 to December 2016 (in-sample period from 2001 to 2015 and out-of-sample consisting of 2016). The second approach considered a rolling window with a constant length of 5-years: 4years data to perform in-sample estimations and an out-of-sample evaluation period of 1-year. In this case, the first window ranges from January 2003 to December 2007 (in-sample period from 2003 to 2006 and out-of-sample consisting of 2007) while the last window ranges from January 2012 to December 2016 (in-sample period from 2012 to 2015 and out-of-sample consisting of 2016).

The application of historical windows of different lengths allows the observation of the effect of considering long-term past data over short-term past data in the definition of the uncertainty set and, therefore, it allows the investigation of whether the use of long-term past data affects the predictive accuracy of the model. Previous studies have shown that long-term past returns (measured over long-term periods) are negatively correlated with future returns, a phenomenon referred to as the long-term reversal effect (Bondt and Thaler, 1985), while short-term past returns (measured over the last year) are positively correlated with future returns, a phenomenon referred to as the momentum effect (Jegadeesh and Titman, 1993).

To explore the sensitivity of the results to the variation of the relative risk aversion parameter, different values $(\gamma \in\{0.5,2,5\})$ were explored and the absolute and relative robust models as well as the classical mean-variance model were solved for each one of these values. It is important to notice that, while the different values of $\gamma$ define different AR, RR and MV portfolios, they do not influence the GMV, EW and WS portfolios (only the corresponding measure of regret).

Adjusted closing prices of the stocks in the constituent list of the DAX index at the end of the insample period were collected and daily continuous returns were calculated.

The steps for computing the absolute and relative-robust solutions, described in the previous section, are iteratively repeated for each of the time windows defined. For each of the time windows under analysis, the random generation of the estimation and validation subsamples and the GA optimization are repeated 100 times $(\mathrm{R}=100)$. A constant length of 1 -year observations was defined for each subsample; hence, the estimation and validation subsamples correspond to a 2-years in-sample period of consecutive daily returns. A total of 100 scenarios $(S=100)$ are computed for each estimation subsample. Each scenario, defined by the sample mean and the 
sample covariance matrix, is computed considering an estimation window length of 120 consecutive daily returns. Estimations of the model inputs are performed in R.

Once the robust and non-robust portfolios are computed for each of the time windows under analysis, in-sample and out-of-sample performances are analyzed.

\subsection{Portfolio performance analysis}

In order to investigate the real contribution of relative robust models within the portfolio optimization field of study, the performance of the proposed relative robust strategy was analyzed and compared to classical non-robust optimization strategies, considering both in-sample and outof-sample data. A comparison to the relative robust strategy presented by Xidonas et al. (2017) was also made which allowed the assessment of the current contribution within the field of relative robustness approaches. Additionally, and since there is a lack of empirical studies that compare the performance of relative-robust and absolute-robust portfolios, the performance of the proposed relative-robust and absolute-robust portfolios is analysed.

The non-robust optimization problems applied were the minimization of the variance subject to a lower limit on the expected return, defined by

$$
\begin{aligned}
& \min x^{T} \sum x \\
& \text { s.t. } x^{T} \mu \geq \mu_{0} \\
& \quad x \in X
\end{aligned}
$$

and the global minimum variance problem, defined by

$$
\begin{aligned}
& \min x^{T} \Sigma x \\
& \text { s.t. } x \in X
\end{aligned}
$$

Problems (15) and (16) were solved and the MV and GMV portfolios were identified. For solving problem (15), first the Markowitz's efficient frontier was constructed considering (all) possible values for $\mu_{0}$ (lower limit on the expected return); then, the MV portfolio was identified by selecting the efficient portfolio that maximizes the investor's expected utility according to the different values of the relative risk aversion parameter. Inputs for the classic models were estimated within the entire in-sample window, namely the in-sample mean and the in-sample covariance matrix were calculated considering 15-years data or 4-years data, according to the approach under consideration. Optimal solutions were computed using CPLEX. The EW portfolio, which equally allocates the wealth by the assets that were included in each of the windows under analysis, was also created.

To replicate the relative robust strategy presented by Xidonas et al. (2017), 5 scenarios corresponding to the average return and mean absolute deviation from 80, 60, 40, 20 and 10weeks historical data were computed. Eleven different weight combinations were also used, namely $(0,1),(0.1,0.9), \ldots,(0.9,0.1),(1,0)$, and, for each window under analysis, eleven relative-robust portfolios were computed using CPLEX. These portfolios will be hereby designated by WS1, WS2, ..., WS10 and WS11, respectively.

After determining RR, MV, GMV, EW and WS1 to WS11 portfolios, in-sample ${ }^{4}$ and out-ofsample performances were compared by analyzing the portfolios annualized return, risk and (modified) Sharpe ratio. The Israelsen's modified Sharpe ratio (Israelsen, 2005) defined by

$S_{I}=\frac{x^{T} \mu-r_{f}}{\sqrt{d x^{T} \Sigma x}\left(\left(x^{T} \mu-r_{f}\right) / a b s\left(x^{T} \mu-r_{f}\right)\right)}$,

where $x^{T} \mu-r_{f}$ represents the annualized excess return of the portfolio comparatively to the return of the risk-free asset $\left(r_{f}\right), d$ corresponds to the number of observations (trading days) in a year and $a b s($.$) is the absolute value function, was used. The risk-free asset selected for the$

\footnotetext{
${ }^{4}$ In the in-sample analysis the overall in-sample period was used in order to compute estimators for the models.
} 
computation of the modified Sharpe ratio was the 1-year maturity government triple A bond for the area accessible at https://www.ecb.europa.eu/stats/financial markets and interest rates/euro area yield curves/h tml/index.en.html. This indicator was only computed for the out-of-sample analysis since data on the risk-free asset is only available from January 2006 onwards. The Israelsen's modified Sharpe ratio $\left(S_{I}\right)$ is equal to the standard Sharpe ratio when excess return is positive while providing correct rankings regardless of the excess return being positive or negative.

In addition, the regret, defined by

$R=\left(\frac{\left(1+x^{s * T} \mu^{s}\right)^{1-\gamma}}{1-\gamma}-\frac{\gamma}{2}\left(1+x^{s * T} \mu^{s}\right)^{-\gamma-1} x^{s * T} \Sigma^{S} x^{S *}\right)-\left(\frac{\left(1+x^{T} \mu^{s}\right)^{1-\gamma}}{1-\gamma}-\frac{\gamma}{2}\left(1+x^{T} \mu^{S}\right)^{-\gamma-1} x^{T} \Sigma^{S} x\right)$

where $x *$ represents the optimal portfolio (feasible solution with maximum utility) within the sample period under consideration, was calculated and compared for the in-sample and out-ofsample periods.

\subsection{Results}

The effect of the variation of both the in-sample period length and the relative risk aversion parameter is analyzed. For that purpose, the composition and the performance of the RR, the MV and the GMV portfolios, are investigated and compared. In particular, the composition of the portfolios concerning the maximum weight of an asset (Max\%), the sum of the 3 maximum weights in the portfolio (Sum3Max\%) and the number of assets with non-zero weights in each computed portfolio (Cardinality) are identified. Mean values obtained over the 10 windows are presented. Since the optimal portfolios have some assets with very small but not necessarily zero weights, we measure cardinality as the number of assets with weights higher than $0.1 \%$. Acknowledging the limitations of using the average return as the sole comparison measure, the portfolios' performances are analyzed, both in-sample and out-of-sample, by comparing the mean of the portfolios' returns (mean return) and the mean of the portfolios' variances (mean risk), obtained over the 10 windows. Additionally, the mean of the portfolios' regrets (mean regret) and the mean of the portfolios' (out-of-sample) modified Sharpe ratio $\left(S_{I}\right)$, obtained over the 10 windows, are also analyzed for all the computed portfolios. The consistency of the portfolios in terms of the proximity to their expected performance is assessed by comparing the in-sample and out-of-sample results. The portfolios' regrets reflect the robustness of the optimal solutions in terms of the utility loss for the investor resulting from choosing a given portfolio instead of choosing the optimal portfolio of the realized scenario for the uncertain parameters.

Then, the composition and the in-sample and out-of-sample performances of relative-robust and non-robust portfolios are compared for each of the 10 windows. Finally, the performances of the $\mathrm{RR}$ and the AR portfolios are compared in each one of the out-of-sample years. Results are presented for the in-sample period length and the values of the risk aversion parameter associated with the best (mean) performances for both in-sample and out-of-sample datasets.

\subsubsection{Effect of the variation of the in-sample period length and the risk aversion parameter}

Table 1 presents the composition of the RR, MV and GMV portfolios by length of the in-sample period and/or the value of the risk aversion parameter considered for their computation. It can be observed that for higher levels of risk aversion and regardless of the in-sample period length, the exposure of the RR and MV portfolios to individual stocks decreases since the mean of the maximum values of the assets' weights (Max\% and Sum3Max\%) decreases and the mean number of assets with non-zero weights (Cardinality) increases.

Table 1: Composition of the RR, MV and GMV portfolios by length of the in-sample period considered for their computation.

\begin{tabular}{lccc}
\hline \multicolumn{1}{c}{ Portfolio } & Max\% & Sum3Max\% & Cardinality \\
\hline RR40.5 & 0.323 & 0.723 & 18 \\
RR42 & 0.215 & 0.519 & 19
\end{tabular}




\begin{tabular}{lccc}
$R R 45$ & 0.144 & 0.353 & 22 \\
$R R 150.5$ & 0.500 & 0.794 & 19 \\
RR152 & 0.168 & 0.422 & 21 \\
RR155 & 0.113 & 0.278 & 23 \\
MV40.5 & 0.893 & 1.000 & 2 \\
MV42 & 0.625 & 0.925 & 4 \\
MV45 & 0.462 & 0.881 & 5 \\
MV150.5 & 0.733 & 0.956 & 3 \\
MV152 & 0.393 & 0.797 & 6 \\
MV155 & 0.251 & 0.611 & 8 \\
GMV4 & 0.263 & 0.600 & 10 \\
GMV15 & 0.175 & 0.470 & 13 \\
\hline
\end{tabular}

This table presents the characteristics of the optimal portfolios that are influenced by the length of the in-sample period and/or the value of the risk aversion parameter considered for their computation. Here, the composition of the portfolios regarding the maximum weight of an asset (Max\%), the sum of the 3 maximum weights in the portfolio (Sum3Max\%), and the number of assets with non-zero weights in each computed portfolio (Cardinality) are described. As explained before, for measuring the cardinality, only the number of assets with weights higher than $0.1 \%$ is considered. The optimal portfolios were represented according to the length of the in-sample period and the value of the risk aversion parameter used in their computation. For instance, 'RR155', 'MV155' and 'GMV15' corresponds, respectively, to the RR, MV and GMV portfolios computed using 15-years data to perform in-sample estimations and, in the case of the RR and the $M V$ portfolios, using a value of 5 for the risk aversion parameter.

Furthermore, when the length of the in-sample period is reduced (from 15 to 4 years), the exposure of the portfolios to individual stocks increases in the three optimal portfolios, since the mean of the maximum values of the assets' weights (Max\% and Sum3Max\%) increases and the mean number of assets with non-zero weights (Cardinality) decreases. This result is verified regardless of the risk aversion parameter's value, except for the RR portfolio computed using a value of 0.5 for the risk aversion parameter; although the cardinality slightly decreases for this portfolio, the mean of the maximum values of the assets' weights decreases when the in-sample length is reduced.

It is also possible to observe that, regardless of the in-sample period length, the MV portfolio is the less diversified portfolio while the RR portfolio is the most diversified one.

The in-sample and the out-of-sample mean risk and mean return of the RR, MV and GMV portfolios are presented in Figure 2. The optimal portfolios were represented according to the length of the in-sample and/or the value of the risk aversion parameter considered in their computation. This means that RR155 represents the relative-robust portfolio computed using an in-sample period of 15 years and a value of 5 for the risk aversion parameter while GMV4 represents the global minimum variance portfolio based on an in-sample period of 4 years, for instance.

Analysing the effect of the length of the in-sample period in the portfolios' performance, it can be observed that when reducing the length of the in-sample period (from 15 years to 4 years), the in-sample mean return and mean risk of the MV portfolio increase. Notice that, while the mean return suffers a substantial increase despite the value of the risk aversion parameter, the increment of the mean risk is more accentuated for lower risk aversion. A similar trend is observed among the RR portfolios. First, reducing the length of the in-sample period (from 15 years to 4 years), increases both the in-sample mean return and mean risk of the RR portfolios. Second, it is quite evident that the length seems to have more substantial effect in the in-sample mean return of the $\mathrm{RR}$ portfolio, since the mean risk is very similar when the risk aversion parameter is 5 or 2 ; for a value of the risk aversion parameter of 0.5 , the overall in-sample performance of the RR portfolio is improved when reducing the length of the in-sample period since the RR40.5 portfolio presents higher mean return and lower mean risk comparatively to the RR150.5 portfolio. Regarding the GMV portfolio, its overall in-sample performance is improved when reducing the length of the in-sample period as the in-sample mean return substantially increases while the in-sample mean risk decreases. 
Except for the parametrization described by an in-sample period of 15 years and a value of 5 for the risk aversion parameter, it can be confirmed that the RR always reveals lower in-sample mean risk comparatively to the corresponding MV portfolio (for a given parametrization). Additionally, the MV portfolio presents the best (highest) in-sample mean return and the GMV portfolio presents the best (lowest) in-sample mean risk.

Concerning the effect of the in-sample period length in the out-of-sample performance of the optimal solutions, it can be observed that, when the length is reduced, the out-of-sample mean return of the RR portfolio is improved, regardless the value of the risk aversion parameter. On the other hand, reducing the length of the in-sample period seems to improve the out-of-sample mean risk when the risk aversion parameter is 5 or 0.5 ; for a value of 2 , the mean risk slightly worsens (from 0.054 to 0.061 , approximately) when the in-sample period length is reduced. It is also important to highlight that the RR152 is the only RR portfolio that presents negative $(-0,010 \%$, very close to $0 \%$ ) out-of-sample mean return.

Analyzing the MV portfolio, its general out-of-sample performance deteriorates when reducing the in-sample period length since the mean return decreases while its mean risk increases, regardless of the value of the risk aversion parameter. It is also quite clear that the overall out-ofsample performance of the MV portfolio is improved when the value of the risk aversion parameter is increased, regardless of the in-sample period length used in its computation. The worst performances are presented by the MV42, MV40.5 and MV150.5 portfolios, which present the lowest (negative) out-of-sample mean returns and the highest out-of-sample mean risks.

An opposite trend is observed for the GMV portfolio, since its out-of-sample mean return increases while its out-of-sample mean risk decreases, revealing an improvement in its overall out-of-sample performance. Here, an unexpected result is found regarding the GMV portfolio: although the exposure of the portfolio to individual stocks increased when reducing the length of the in-sample period, as previously verified (Table 1), both the in-sample and the out-of-sample mean risks of the GMV portfolio have decreased. A similar behaviour can be observed by the RR portfolios computed using a risk aversion parameter of 5 or 0.5 ; for the remaining portfolios, the decrease in the diversification level caused, as expected, an increase in their mean risk.

It is important to highlight that, when reducing the length of the in-sample period, the RR becomes a dominant solution when compared to the corresponding MV portfolio since it presents better out-of-sample performance in all the performance measures considered for the analysis. Furthermore, regardless of the length of the in-sample period and the value of the risk aversion parameter, the GMV portfolio is a dominant solution when compared to the RR and the MV portfolio, presenting better out-of-sample performance in all the performance measures under consideration. This result is in accordance with previous studies (Chan, Karceski and Lakonishok, 1999; Jagannathan and Ma, 2003) supporting the outperformance of the GMV portfolio. 


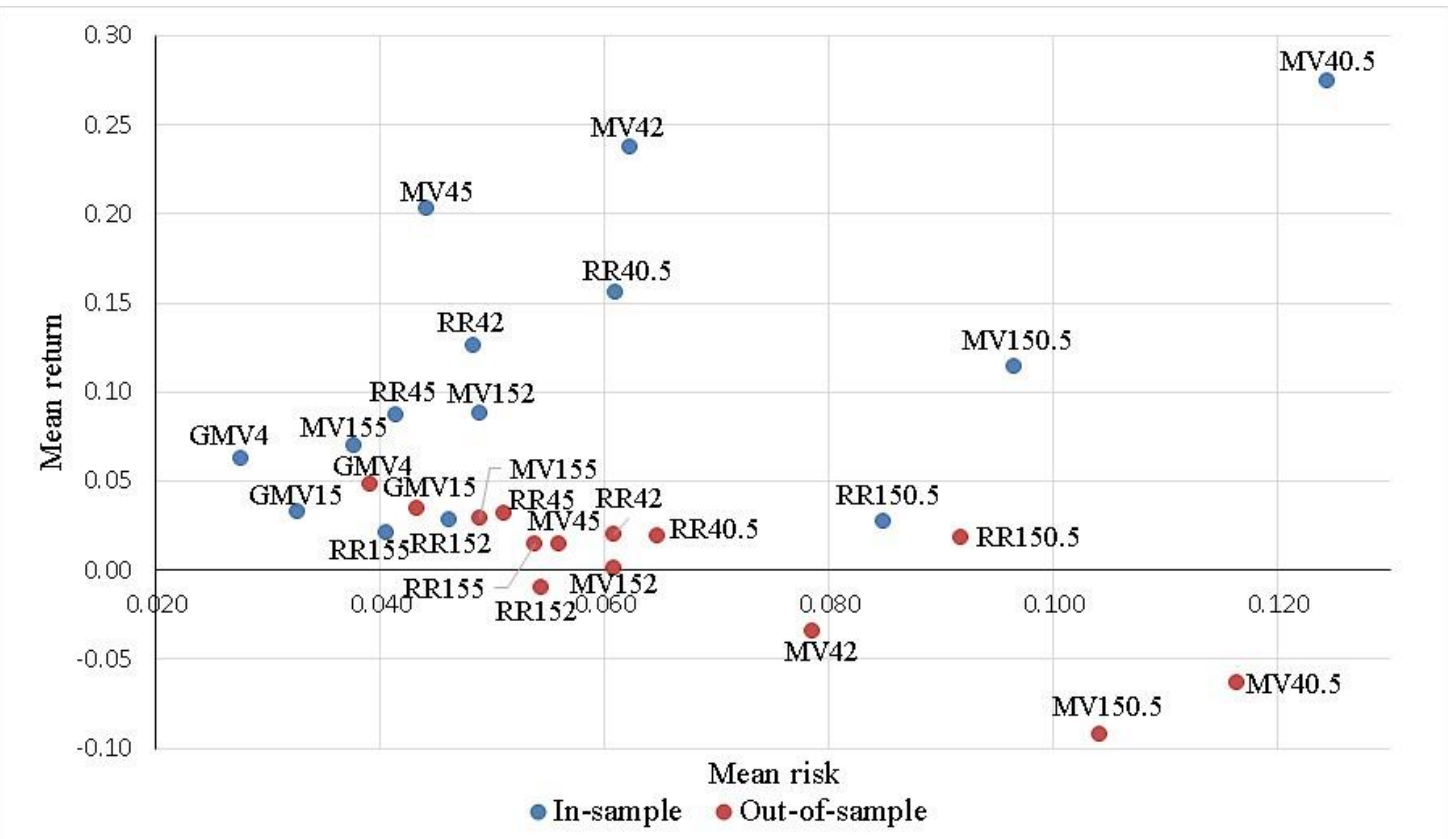

Figure 2: In-sample and out-of-sample mean risk and mean return of the RR, MV, GMV portfolios. The optimal portfolios were represented according to the length of the in-sample period and the value of the risk aversion parameter used in their computation. For instance, 'RR155', 'MV155' and 'GMV15' corresponds, respectively, to the RR, MV and GMV portfolios computed using 15-years data to perform in-sample estimations and, in the case of the RR and the $M V$ portfolios, using a value of 5 for the risk aversion parameter.

The distances between in-sample and out-of-sample portfolios' locations can be observed in Figure 2, allowing to draw some conclusions about the proximity to the expected performance of the portfolios. The portfolios that present the smallest distances between in-sample and out-ofsample mean return and mean risk and, therefore, are more consistent concerning the proximity to the expected performance, are the GMV portfolio (regardless of the length of the in-sample period) and RR portfolio computed using the in-sample period length of 15 years (regardless of the risk aversion parameter). Thus, the length of the in-sample period seems to have no significant effect on the proximity to the expected performance of these portfolios.

The same cannot be stated for the MV portfolio and the RR portfolio computed using an in-sample period length of 4 years. The proximity to the expected performance of the optimal portfolio RR seems to be negatively affected when the length of the in-sample period is reduced, especially when mean returns are considered. It is also important to highlight that the proximity to the expected performance of the RR portfolio computed using an in-sample period length of 4 years seems to be improved when higher levels of risk aversion are considered.

Concerning the MV portfolio, it presents the largest distances between in-sample and out-ofsample portfolio locations, with the higher distance occurring for the MV computed based on an in-sample period of 4 years (regardless of the risk aversion parameter). Thus, reducing the length of the in-sample period from 15 to 4 years seems to negatively affect the proximity to the expected performance of the MV solution. Also, and similar to the RR portfolio computed based on an insample period of 4 years, increasing the value of the risk aversion parameter seems to positively affect the proximity to the expected performance of the MV solution since the distances between in-sample and out-of-sample mean return and mean risk decrease.

The out-of-sample (OS) performances of the optimal relative-robust and non-robust portfolios concerning regret and modified Sharpe ratio $\left(S_{I}\right)$ are described in Table 2. The results are presented according to the in-sample period length and for a risk aversion parameter equal to 5 . Even though the results are not presented for the remaining values of the risk aversion parameter, the conclusions are valid for all the parametrizations considered. Notice that, the computation of the EW and WS portfolios is not influenced by the variation of the in-sample period length or the risk aversion parameter. The values presented correspond to the mean of the portfolios' regrets and mean of the portfolios $S_{I}$, obtained over the 10 time windows. 
Table 2: Out-of-sample regret and modified Sharpe ratio $\left(S_{I}\right)$ of the $R R, M V, G M, E W$ and WS portfolios by length of the in-sample period and considering a value of 5 for the risk aversion parameter.

\begin{tabular}{ccccc}
\hline \multirow{2}{*}{ Portfolio } & \multicolumn{2}{c}{ OS Regret } & \multicolumn{2}{c}{ OS $S_{I}$} \\
\cline { 2 - 4 } & 15Years & 4Years & 15Years & 4Years \\
\hline RR & 0.25350 & 0.19833 & 0.48734 & 0.57761 \\
MV & 0.20004 & 0.21983 & 0.48688 & 0.43926 \\
GMV & 0.18176 & 0.15336 & 0.53089 & 0.54749 \\
EW & 0.32404 & 0.40703 \\
WS1 & 0.40421 & 0.32463 \\
WS2 & 0.40888 & 0.32534 \\
WS3 & 0.40727 & 0.33024 \\
WS4 & 0.37656 & 0.40022 \\
WS5 & 0.29309 & 0.38958 \\
WS6 & 0.23415 & 0.32499 \\
WS7 & 0.19839 & 0.36022 \\
WS8 & 0.18222 & 0.41041 \\
WS9 & 0.17916 & 0.44457 \\
WS10 & 0.19039 & 0.48574 \\
WS11 & 0.19346 & 0.52163 \\
\hline
\end{tabular}

This table presents the out-of-sample (OS) performances of the optimal relative-robust and non-robust portfolios concerning regret and modified Sharpe ratio $\left(S_{I}\right)$ by length of the in-sample period and considering a value of 5 for the risk aversion parameter. The values presented correspond to the mean of the portfolios' regrets and mean of the portfolios $S_{I}$, obtained over the 10 time windows. For computing the mean regret, the worst result was not considered since it was verified an atypical regret value (twenty to two hundred times higher than the highest of the remaining values) in the out-of-sample year of 2008.

It can be observed that reducing the in-sample period length leads to lower levels of out-of-sample mean regret for the RR and GMV portfolios. In the case of the MV portfolio the mean regret has a slight increase. Concerning the mean $S_{I}$, the results confirm that reducing the in-sample period length seems to substantially improve the performance of the RR and GMV portfolios, while deteriorating the overall out-of-sample performance of the MV portfolio.

Regarding the remaining optimal solutions, it can be verified that the EW portfolio shows the highest out-of-sample mean regret comparatively to the remaining non-robust portfolios. Furthermore, the WS portfolios corresponding to lower $w_{1}^{g}$ weights (when maximizing return is weighted more than minimizing risk), namely WS1, WS2, WS3 e WS4, underperform comparatively to WS portfolios corresponding to higher $w_{1}^{g}$ weights (when minimizing risk is weighted more than maximizing return), namely WS7, WS8, WS9, WS10 and WS11, since they reveal higher mean regrets and lower mean $S_{I}$. Moreover, out-of-sample mean regrets seem to support the results found by Xidonas et al. (2017), since the WS portfolios corresponding to higher $w_{1}^{g}$ weights are more robust, in terms of utility loss for the investor, relative to the WS portfolios corresponding to lower $w_{1}^{g}$ weights.

Finally, it is important to outline some relevant results based on an in-sample period of 4 years. The RR presents itself as a more robust solution than the MV, the EW and the majority of the WS portfolios, in terms of utility loss for the investor, since it shows a lower out-of-sample mean regret. Comparing the out-of-sample mean $S_{I}$ of all computed portfolios, it is possible to observe that the RR, GMV and WS11 portfolios show similar performances, with the best performance achieved by the RR portfolio. On the contrary, the worst performances are from the EW portfolio and the WS portfolios, corresponding to lower $w_{1}^{g}$ weights.

The analysis of the in-sample and the out-of-sample performances of relative-robust and nonrobust portfolios, for each of the 10 windows, is presented next. Since the RR portfolio, as well as the GMV portfolio, generally present better performances for the in-sample period length 
corresponding to 4 years of historical data and/or a value of 5 for the risk aversion parameter, results are described for this particular case. The results that will be presented in the next section generally prevail regardless of the length of the in-sample period and the risk aversion parameter.

\subsubsection{Performance of relative-robust and non-robust portfolios}

Before analyzing the performance of the optimal portfolios, it is important to examine their characteristics concerning composition in order to identify similarities as well as the main differences between robust and non-robust solutions. The results are shown in Table 3 .

The RR portfolio reveals a minimum cardinality of 19 and a maximum of 27, while it presents a maximum asset weight varying between $10 \%$ and $35 \%$, and a maximum investors' wealth allocation to 3 assets varying between $25 \%$ and $61 \%$.

The MV portfolio presents a low diversification level since its cardinality is between 3 and 7, it has a maximum asset weight varying between $23 \%$ and $68 \%$ and, for most of the windows, it allocates $81 \%$ or more of the investors' wealth to only 3 assets. Comparatively to the MV portfolio, the GMV portfolio is more diversified, presenting a minimum cardinality of 8 and a maximum of 12, and a lower exposure to individual stocks, since the maximum weight of an asset varies between $18 \%$ and $34 \%$ while the sum of the 3 maximum weights varies between $48 \%$ and $70 \%$.

Regarding WS portfolios, it can be verified that as the $w_{1}^{g}$ weights increase, the level of portfolio diversification also increases, since the exposure to individual stocks decreases. These results were expected since the highest levels of the $w_{1}^{g}$ weights correspond to portfolios that mainly aim to maximize the expected return while the lowest levels of the $w_{1}^{g}$ weights correspond to portfolios that mainly aim to minimize the mean absolute deviation. Thus, the WS portfolios with the lowest $w_{1}^{g}$ weights are highly concentrated (cardinality between 2 and 5), similarly to the MV portfolio, with exposure to a single asset higher than $50 \%$ and a cumulative exposure to three assets equal to $100 \%$, in most of the windows. On the other hand, the WS portfolios with the highest $w_{1}^{g}$ weights are more diversified (cardinality between 6 and 15), similarly to the GMV portfolio, and reveal lower exposure to individual stocks, since the maximum weight of an asset (sum of the 3 maximum weights) varies between $16 \%$ and $62 \%$ ( $45 \%$ and $86 \%$ ).

Table 3: Composition of the optimal portfolios regarding the maximum weight of an asset (Max\%), sum of the 3 maximum weights (Sum3Max\%) and number of assets with weights higher than $0.1 \%$ (Card.) for each computed portfolio and by window.

\begin{tabular}{|c|c|c|c|c|c|c|c|c|c|c|}
\hline & & $\mathrm{RR}$ & MV & EW & GMV & WS1 & WS2 & $\ldots \quad$ WS5 & $\ldots \quad$ WS10 & WS11 \\
\hline \multirow{3}{*}{ 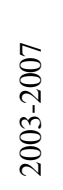 } & $\operatorname{Max} \%$ & 0.16 & 0.68 & 0.05 & 0.34 & 0.65 & 0.71 & 0.69 & 0.34 & 0.34 \\
\hline & Sum3Max \% & 0.41 & 1 & 0.15 & 0.70 & 1 & 1 & 1 & 0.59 & 0.59 \\
\hline & Card. & 19 & 3 & 20 & 10 & 2 & 2 & 3 & 11 & 11 \\
\hline \multirow{3}{*}{ 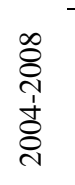 } & $\operatorname{Max} \%$ & 0.35 & 0.41 & 0.05 & 0.21 & 0.52 & 0.42 & 0.49 & 0.28 & 0.25 \\
\hline & Sum3Max \% & 0.61 & 0.93 & 0.16 & 0.48 & 1 & 0.87 & 0.82 & 0.66 & 0.67 \\
\hline & Card. & 19 & 5 & 19 & 11 & 3 & 4 & 6 & 12 & 11 \\
\hline \multirow{3}{*}{$\begin{array}{l}\stackrel{8}{8} \\
\text { ì } \\
\grave{o} \\
\stackrel{d}{0}\end{array}$} & $\operatorname{Max} \%$ & 0.12 & 0.35 & 0.05 & 0.28 & 0.42 & 0.43 & 0.51 & 0.33 & 0.28 \\
\hline & Sum3Max\% & 0.31 & 0.81 & 0.15 & 0.60 & 1 & 1 & 0.99 & 0.76 & 0.73 \\
\hline & Card. & 20 & 5 & 20 & 9 & 3 & 3 & 4 & 8 & 8 \\
\hline \multirow{3}{*}{ 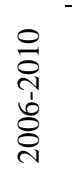 } & $\operatorname{Max} \%$ & 0.11 & 0.34 & 0.05 & 0.27 & 0.78 & 0.73 & 0.41 & 0.31 & 0.32 \\
\hline & Sum3Max\% & 0.31 & 0.75 & 0.14 & 0.55 & 1 & 1 & 0.79 & 0.71 & 0.73 \\
\hline & Card. & 21 & 6 & 21 & 8 & 2 & 3 & 6 & 10 & 8 \\
\hline \multirow{2}{*}{$\frac{1}{8} \bar{c}$} & $\operatorname{Max} \%$ & 0.10 & 0.23 & 0.04 & 0.25 & 0.78 & 0.76 & 0.34 & 0.28 & 0.27 \\
\hline & Sum3Max\% & 0.27 & 0.63 & 0.13 & 0.57 & 1 & 1 & 0.75 & 0.63 & 0.56 \\
\hline
\end{tabular}




\begin{tabular}{|c|c|c|c|c|c|c|c|c|c|}
\hline Card. & 22 & 7 & 23 & 10 & 2 & 2 & 6 & 9 & 10 \\
\hline $\operatorname{Max} \%$ & 0.13 & 0.64 & 0.04 & 0.27 & 0.78 & 0.82 & 0.64 & 0.32 & 0.31 \\
\hline Sum3Max \% & 0.37 & 0.90 & 0.12 & 0.67 & 1 & 1 & 0.93 & 0.81 & 0.76 \\
\hline Card. & 26 & 5 & 26 & 11 & 2 & 2 & 4 & 6 & 7 \\
\hline Max\% & 0.12 & 0.35 & 0.04 & 0.27 & 0.59 & 0.60 & 0.35 & 0.26 & 0.28 \\
\hline Sum3Max $\%$ & 0.36 & 0.96 & 0.13 & 0.63 & 1 & 1 & 0.81 & 0.60 & 0.65 \\
\hline Card. & 22 & 4 & 24 & 9 & 3 & 3 & 5 & 10 & 8 \\
\hline $\operatorname{Max} \%$ & 0.12 & 0.55 & 0.04 & 0.26 & 0.73 & 0.76 & 0.37 & 0.20 & 0.26 \\
\hline Sum3Max\% & 0.31 & 0.92 & 0.12 & 0.62 & 1 & 1 & 0.74 & 0.53 & 0.56 \\
\hline Card. & 23 & 5 & 26 & 9 & 2 & 2 & 6 & 15 & 13 \\
\hline $\operatorname{Max} \%$ & 0.12 & 0.51 & 0.04 & 0.31 & 0.95 & 0.97 & 0.66 & 0.29 & 0.27 \\
\hline Sum3Max \% & 0.32 & 0.91 & 0.12 & 0.68 & 1 & 1 & 0.90 & 0.68 & 0.68 \\
\hline Card. & 20 & 5 & 26 & 10 & 2 & 2 & 6 & 9 & 9 \\
\hline $\operatorname{Max} \%$ & 0.11 & 0.57 & 0.04 & 0.18 & 0.69 & 0.67 & 0.23 & 0.60 & 0.62 \\
\hline Sum3Max \% & 0.25 & 1 & 0.11 & 0.49 & 1 & 1 & 0.66 & 0.86 & 0.83 \\
\hline Card. & 27 & 3 & 28 & 12 & 2 & 2 & 6 & 7 & 8 \\
\hline
\end{tabular}

Therefore, and comparatively to all the optimal solutions with the exception of the EW portfolio, the RR portfolio has the lowest maximum asset weight and the lowest maximum wealth allocation to 3 assets, revealing itself as the optimal solution with lower exposure to individual stocks. Regarding cardinality, similar results can be observed when comparing the RR and the EW portfolios, suggesting that the proposed methodology tends to assign non-zero weights to the majority of the assets. It is important to highlight that these results are not in accordance with the results concerning the absolute-robust portfolios described in the literature (Kim, Kim and Fabozzi, 2013, 2014).

Figure 3 shows the in-sample and out-of-sample risks and returns of all the optimal portfolios by time window under analysis. Out-of-sample modified Sharpe ratios $\left(S_{\mathrm{I}}\right)$ are presented in Table 4. By analyzing the results for the non-robust portfolios, it can be confirmed that the classical meanvariance strategy reveals conflicting results since it stands out, simultaneously, as the solution with best in-sample performance, while it is among the portfolios with worst out-of-sample performances, in many of the time windows. In fact, for some windows (2009-2013; 2011-2015) the MV portfolio is among those with the highest $S_{I}$, while for others (2004-2008; 2008-2012; 2012-2016) is among those with the lowest $S_{I}$. These results support previous findings concerning the sensitivity of the MV portfolio to the estimation error and the effects of the input uncertainty in the optimization process (Best and Grauer, 1991b; Chopra and Ziemba, 1993; Jagannathan and Ma, 2003; DeMiguel, Garlappi and Uppal, 2009).

Regarding the EW portfolio, a similar conclusion can be drawn, since it is among the portfolios with the highest $S_{I}$ in some windows $(2003-2007$; 2005-2009; 2006-2010; 2008-2012) while it presents poor out-of-sample performance in others $(2007-2011 ; 2010-2014 ; 2011-2015 ; 2012-$ 2016). It is also important to highlight that the EW portfolio outperforms the MV portfolio, in terms of risk and/or in terms of return, in most of the time windows under analysis.

Concerning the GMV portfolio, although this portfolio shows worse in-sample mean return comparatively to the MV portfolio, a different trend can be observed when comparing out-ofsample performances. In fact, the GMV portfolio is a dominant ${ }^{5}$ solution comparatively to the MV and the EW portfolios in the majority of the time windows. Its better performance, relatively to the MV portfolio, can be explained by the fact that its computation requires only the estimates of variances and covariances of the asset returns, becoming less vulnerable to estimation error.

\footnotetext{
${ }^{5}$ A portfolio is considered a dominant solution when it presents, simultaneously, higher return and lower risk.
} 
Furthermore, this portfolio is among the solutions with the best $S_{I}$ in some windows (2004-2008; 2005-2009; 2007-2011; 2008-2012; and 2011-2015) while it is located at the left side of the scatter-plot in all windows, indicating low levels of risk.

Analyzing the WS portfolios, some unexpected outcomes are observed. Although the WS portfolios corresponding to the lowest $w_{1}^{g}$ weights generally present better in-sample returns comparatively to the WS portfolios corresponding to the highest $w_{1}^{g}$ weights, the out-of-sample results show a different picture. Notice that, in the majority of the windows, the WS portfolios corresponding to the lowest $w_{1}^{g}$ weights (WS1, WS2, WS3 and WS4) present lower $S_{I}$ than the WS portfolios corresponding to the highest $w_{1}^{g}$ weights (WS6, WS7, WS8, WS9, WS10 and WS11). Additionally, in some windows (2003-2007 and 2005-2009), the WS1, WS2, WS3, WS4 and WS5 portfolios are the only ones that present negative out-of-sample returns. On the other hand, WS10 and WS11 portfolios show negative out-of-sample returns in 2006-2010 and 20122016, and are the only portfolios with negative out-of-sample returns in 2006-2010. These findings suggest lack of consistency in the out-of-sample performance of the relative robust methodology presented by Xidonas et al. (2017).

In this sense, the authors consider that the proposed relative robust approach stands out as a solid relative robust methodology. First of all, we notice that the RR portfolio provides negative outof-sample returns in time windows characterized by out-of-sample periods of high volatility (the variance of returns of individual assets for the out-of-sample years is shown in Figure 4) and, simultaneously, low mean returns (the mean return of individual assets for the out-of-sample years is shown in Figure 5). Specifically, the underperformance of the RR portfolio occurs in 20042008, 2007-2011 and 2012-2016, where the majority of the optimal portfolios perform poorly.
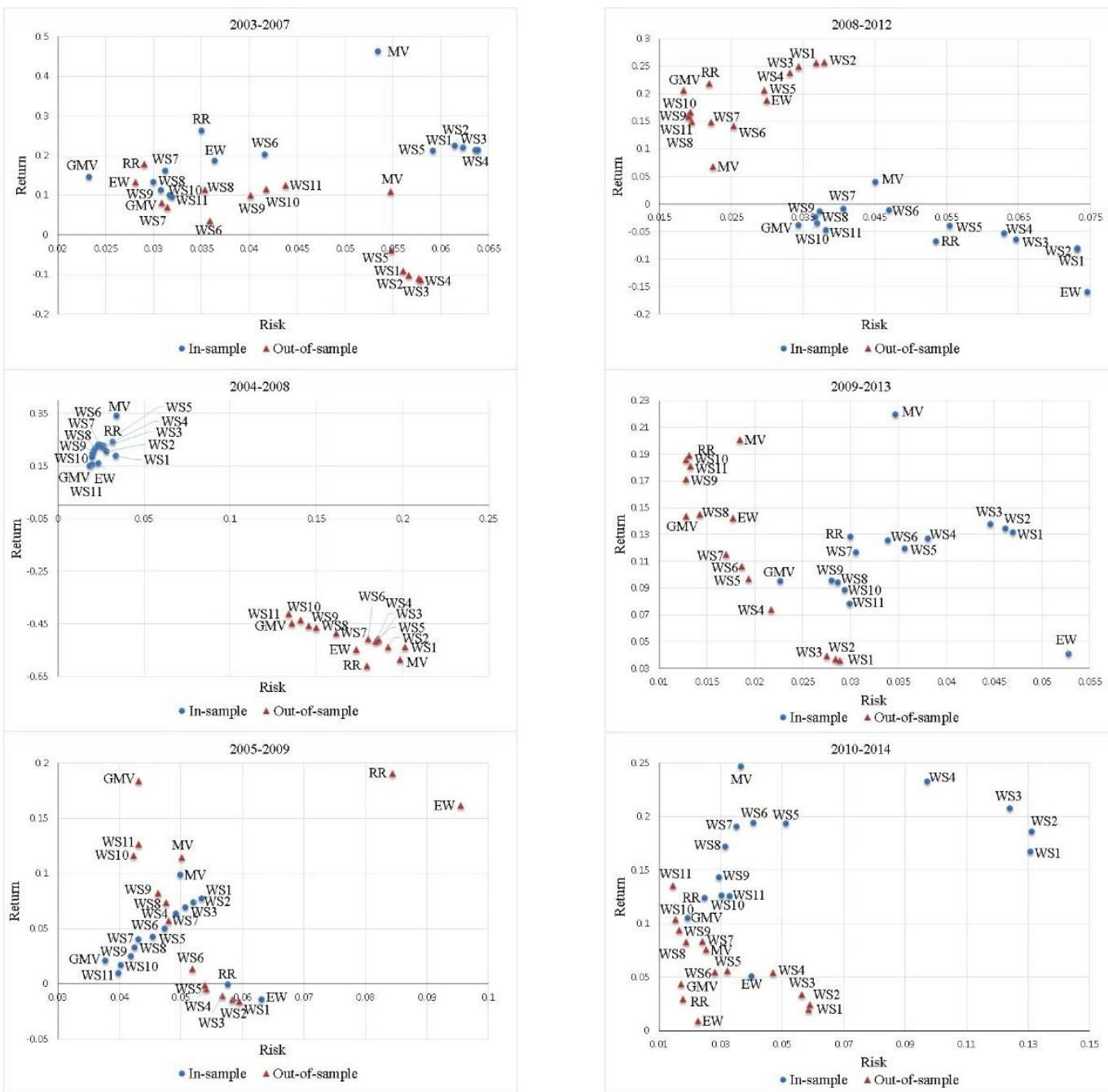

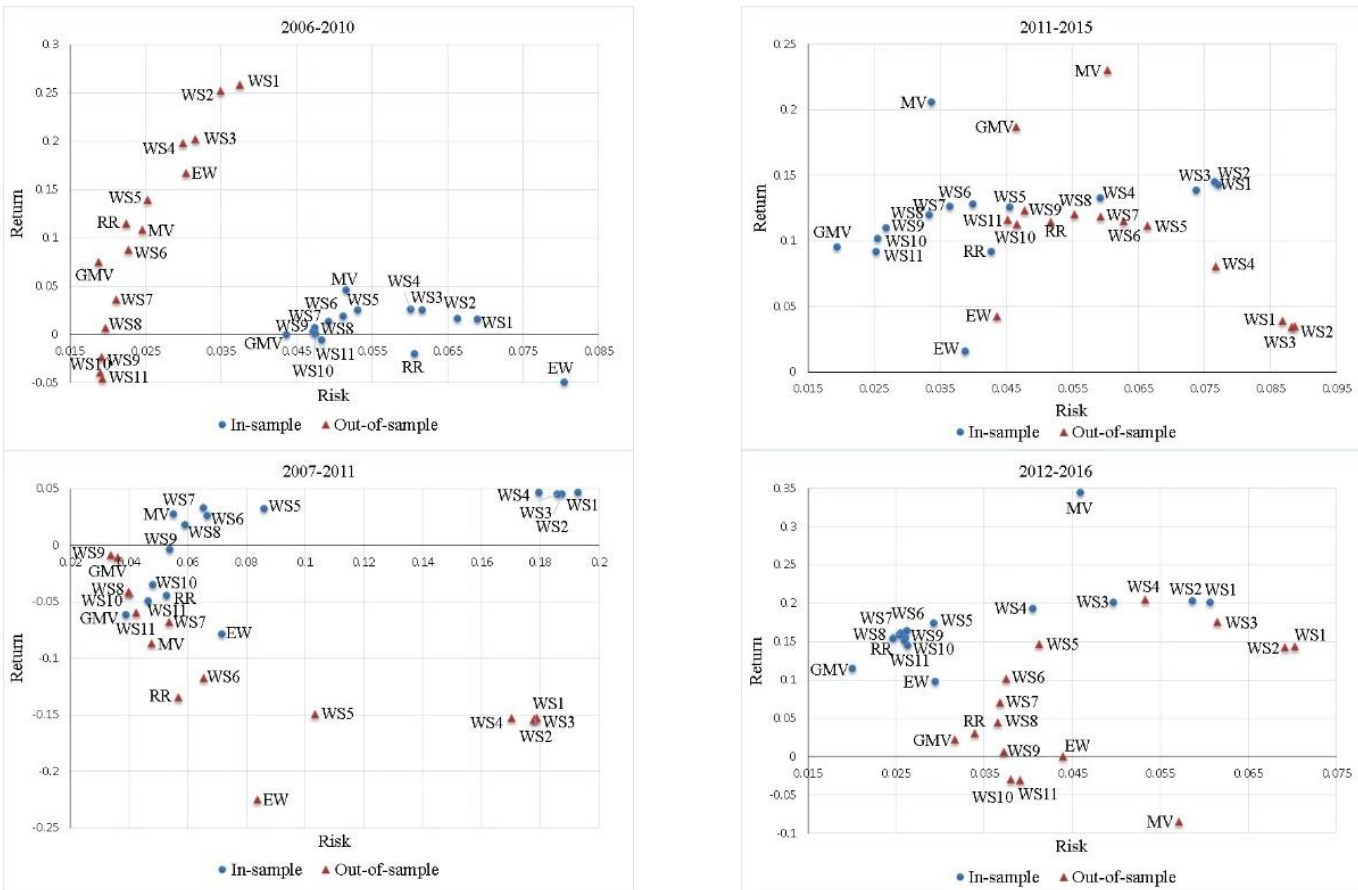

Figure 3: In-sample and out-of-sample risks and returns of all the portfolios computed for each window and considering an in-sample period length of 4 years and a value of 5 for the risk aversion parameter. 


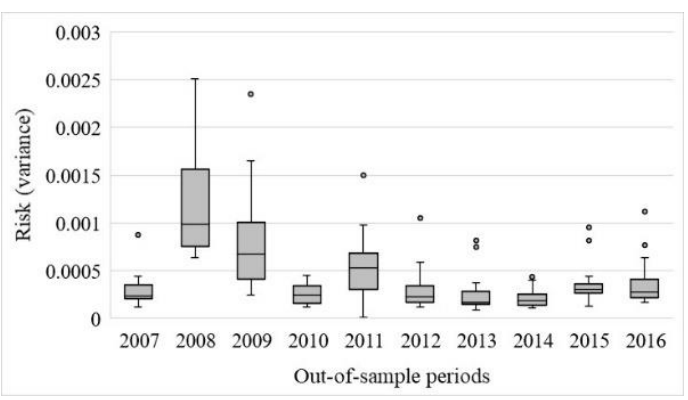

Figure 4: Out-of-sample risk (variance) of individual assets, computed from daily returns.

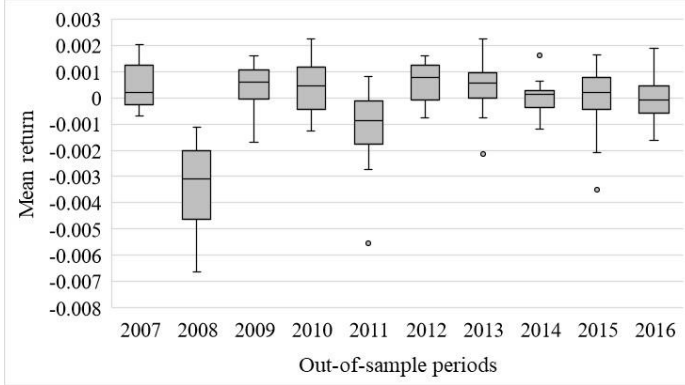

Figure 5: Out-of-sample mean return of individual assets, computed from daily returns.

Secondly, the RR portfolio is located at the left side of the scatter-plot, among the optimal solutions with the lowest risk and it even shows lower risk in out-of-sample data than in in-sample data, in many of the windows under analysis. Considering the $S_{I}$ measure and the out-of-sample results, the RR solution outperforms some of the WS portfolios in 9 of the 10 windows, and it always outperforms the WS portfolios in 3 of the 10 windows. It outperforms the MV portfolio in 7 of the 10 windows, the EW portfolio in 7 of the 10 windows and the GMV portfolio in 3 of the 10 windows. These results clearly reinforce the relevance of the proposed methodology, since previous studies confirmed the good performances of both the $1 / N$ benchmark (DeMiguel, Garlappi and Uppal, 2009) and the GMV portfolio (Chan, Karceski and Lakonishok, 1999; Jagannathan and Ma, 2003).

Furthermore, the out-of-sample return of the RR portfolio is higher than its in-sample return in several windows under analysis. In fact, the tendency of higher out-of-sample returns comparatively to in-sample returns can be explained by the evolution of the stock market, in particular of the DAX index during the out-of-sample periods. The positive evolution of the DAX index during the out-of-sample years is quite evident in Figure 6. Notice that, although the appreciation trend generally prevails during this period, it can be observed severe market drops in 2008 (due to the subprime mortgage crisis) and in 2011 (mainly caused by the European debt crisis). It is also possible to notice that 2015 was a year characterized by high volatility of the DAX index prices as a consequence of severe drops followed by significant recoveries.

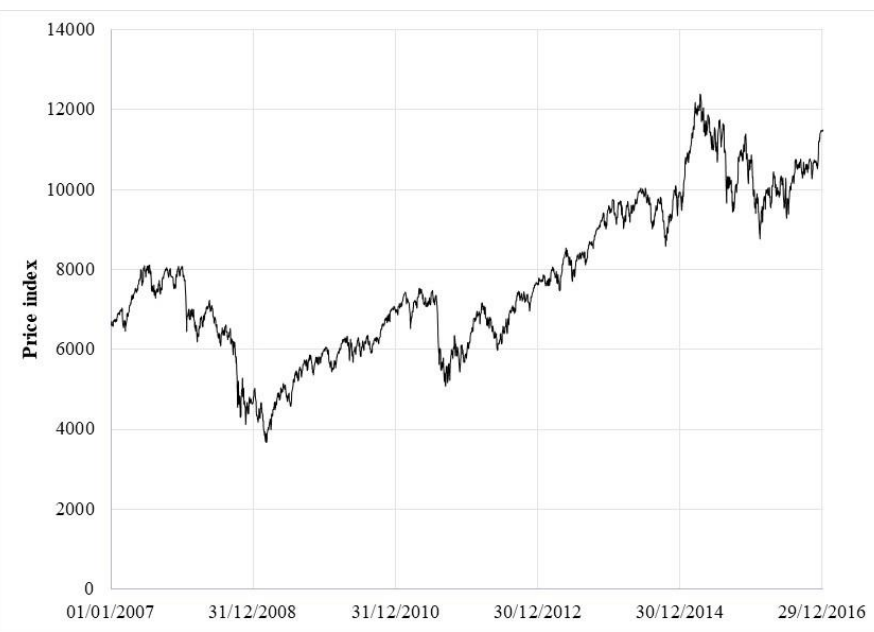

Figure 6: Evolution of the DAX index price during the out-of-sample periods.

The MV portfolio and some of the WS portfolios corresponding to the lowest $w_{1}^{g}$ weights do not reveal the propensity to higher out-of-sample returns comparatively to in-sample returns, which reinforces the underperformance of these portfolios even in favorable market conditions.

Figure 3 also allows the observation of the proximity to the expected performance of the optimal portfolios by analyzing the distances between in-sample and out-of-sample portfolios' location. Although none of the portfolios systematically reveals greater proximity to the expected 
performance in all the windows under analysis, it can be observed that, in many of these windows, the MV and the WS portfolios corresponding to the lowest $w_{1}^{g}$ weights (WS1, WS2, WS3 and WS4) stand out as the solutions that exhibit the highest deviations to their expected performances and, thus, are the less consistent solutions.

It can also be observed that, while the RR portfolio exhibits similar deviations to its expected performance comparatively to the other portfolios in many time windows, in those where the deviations from the expected performance are substantially larger, the RR portfolio stands out as one of the optimal solutions with highest out-of-sample return.

Analyzing the robustness in terms of the utility loss for the investor (Table 5), it can be observed that the RR portfolio is systematically more robust than the EW and the majority of the WS portfolios, revealing lower regrets in most of the windows. Additionally, the RR portfolio is more robust than the MV and the GMV portfolios in 5 of the 10 windows.

Table 4: Out-of-sample modified Sharpe ratio $\left(S_{I}\right)$ of the computed portfolios (by out-of-sample year)

\begin{tabular}{ccccccccccc}
\hline Portfolio & 2007 & 2008 & 2009 & 2010 & 2011 & 2012 & 2013 & 2014 & 2015 & 2016 \\
\hline RR & 0.825 & -0.275 & 0.594 & 0.712 & -0.033 & 1.474 & 1.572 & 0.212 & 0.508 & 0.188 \\
MV & 0.306 & -0.279 & 0.432 & 0.636 & -0.020 & 0.447 & 1.482 & 0.468 & 0.940 & -0.019 \\
EW & 0.571 & -0.244 & 0.465 & 0.910 & -0.067 & 1.079 & 1.073 & 0.053 & 0.206 & 0.024 \\
GMV & 0.245 & -0.179 & 0.800 & 0.484 & -0.003 & 1.517 & 1.269 & 0.322 & 0.872 & 0.148 \\
WS1 & -0.030 & -0.259 & -0.008 & 1.294 & -0.067 & 1.331 & 0.214 & 0.076 & 0.136 & 0.559 \\
WS2 & -0.033 & -0.252 & -0.008 & 1.303 & -0.067 & 1.317 & 0.220 & 0.096 & 0.121 & 0.557 \\
WS3 & -0.035 & -0.239 & -0.007 & 1.090 & -0.067 & 1.341 & 0.240 & 0.137 & 0.119 & 0.724 \\
WS4 & -0.036 & -0.237 & -0.005 & 1.095 & -0.065 & 1.304 & 0.505 & 0.245 & 0.293 & 0.905 \\
WS5 & -0.018 & -0.235 & -0.004 & 0.823 & -0.050 & 1.197 & 0.700 & 0.305 & 0.435 & 0.743 \\
WS6 & 0.000 & -0.232 & -0.001 & 0.526 & -0.032 & 0.880 & 0.781 & 0.319 & 0.461 & 0.548 \\
WS7 & 0.181 & -0.210 & 0.180 & 0.189 & -0.017 & 0.987 & 0.884 & 0.531 & 0.490 & 0.388 \\
WS8 & 0.410 & -0.195 & 0.256 & 0.000 & -0.010 & 1.064 & 1.216 & 0.594 & 0.514 & 0.255 \\
WS9 & 0.310 & -0.189 & 0.300 & -0.004 & -0.003 & 1.170 & 1.518 & 0.723 & 0.567 & 0.054 \\
WS10 & 0.383 & -0.178 & 0.479 & -0.007 & -0.009 & 1.193 & 1.644 & 0.831 & 0.527 & -0.005 \\
WS11 & 0.418 & -0.165 & 0.524 & -0.007 & -0.013 & 1.146 & 1.652 & 1.115 & 0.551 & -0.005 \\
\hline
\end{tabular}

Table 5: Out-of-sample regret of the computed portfolios (by out-of-sample year)

\begin{tabular}{ccccccccccc}
\hline Portfolio & 2007 & 2008 & 2009 & 2010 & 2011 & 2012 & 2013 & 2014 & 2015 & 2016 \\
\hline RR & 0.108 & 132.315 & 0.126 & 0.159 & 0.599 & 0.066 & 0.109 & 0.204 & 0.168 & 0.247 \\
MV & 0.190 & 103.397 & 0.155 & 0.167 & 0.383 & 0.166 & 0.104 & 0.171 & 0.091 & 0.552 \\
EW & 0.136 & 51.794 & 0.162 & 0.133 & 1.476 & 0.088 & 0.135 & 0.238 & 0.236 & 0.313 \\
GMV & 0.183 & 10.449 & 0.093 & 0.186 & 0.174 & 0.069 & 0.129 & 0.188 & 0.106 & 0.253 \\
WS1 & 0.567 & 52.260 & 0.357 & 0.091 & 1.510 & 0.060 & 0.244 & 0.306 & 0.325 & 0.179 \\
WS2 & 0.605 & 49.858 & 0.350 & 0.092 & 1.517 & 0.060 & 0.242 & 0.298 & 0.337 & 0.179 \\
WS3 & 0.636 & 36.598 & 0.340 & 0.114 & 1.519 & 0.061 & 0.237 & 0.278 & 0.337 & 0.144 \\
WS4 & 0.655 & 33.841 & 0.320 & 0.114 & 1.451 & 0.065 & 0.192 & 0.232 & 0.243 & 0.117 \\
WS5 & 0.419 & 32.160 & 0.314 & 0.145 & 0.975 & 0.078 & 0.169 & 0.203 & 0.191 & 0.144 \\
WS6 & 0.241 & 31.699 & 0.283 & 0.181 & 0.574 & 0.112 & 0.161 & 0.197 & 0.182 & 0.176 \\
WS7 & 0.195 & 20.804 & 0.213 & 0.228 & 0.351 & 0.104 & 0.152 & 0.162 & 0.174 & 0.206 \\
WS8 & 0.159 & 14.707 & 0.193 & 0.258 & 0.243 & 0.100 & 0.130 & 0.155 & 0.167 & 0.235 \\
WS9 & 0.179 & 12.848 & 0.181 & 0.298 & 0.163 & 0.092 & 0.113 & 0.142 & 0.155 & 0.288 \\
WS10 & 0.167 & 9.241 & 0.143 & 0.321 & 0.240 & 0.090 & 0.106 & 0.133 & 0.163 & 0.350 \\
WS11 & 0.161 & 5.984 & 0.135 & 0.333 & 0.289 & 0.094 & 0.105 & 0.111 & 0.158 & 0.355 \\
\hline
\end{tabular}


Regarding the WS portfolios, it can be observed that out-of-sample results do not corroborate the conclusion presented by Xidonas et al. (2017), which stated that the area of the Pareto front that corresponds to minimizing risk against maximizing return (portfolios with highest $w_{1}^{g}$ weights), provides more robust solutions. In fact, the WS portfolios with lowest $w_{1}^{g}$ weights are more robust (presenting the lowest regrets) in the sample periods 2006-2010, 2008-2012 and 2012-2016, while the WS portfolios with highest $w_{1}^{g}$ weights reveal the lowest regrets in the remaining time windows. This underlines the relevance of the out-of-sample performance analysis of new portfolio optimization models.

To conclude, is also important to highlight that the GMV and the RR portfolios are among the portfolios with the lowest regrets in the majority of the time windows under analysis, which, in our opinion, validates the proposed relative robust methodology, supporting its robustness.

\subsubsection{Performance of relative-robust and absolute-robust portfolios}

As previously mentioned, there is lack of empirical studies that compare the performance of relative-robust and absolute-robust portfolio models. For that reason, the performance of the proposed RR and AR portfolios were also compared in each of the out-of-sample years and the main results are now presented for the same parametrization (in-sample period length of 4 years and a value of 5 for the risk aversion parameter).

Figure 7 shows the out-of-sample risks and returns of the RR and AR portfolios by out-of-sample year under analysis. The proximity between the RR and AR portfolios' location in the risk/return space makes quite clear that the RR and AR portfolios reveal similar performances in the majority of the out-of-sample years. Although similar, outstanding differences can be observed.

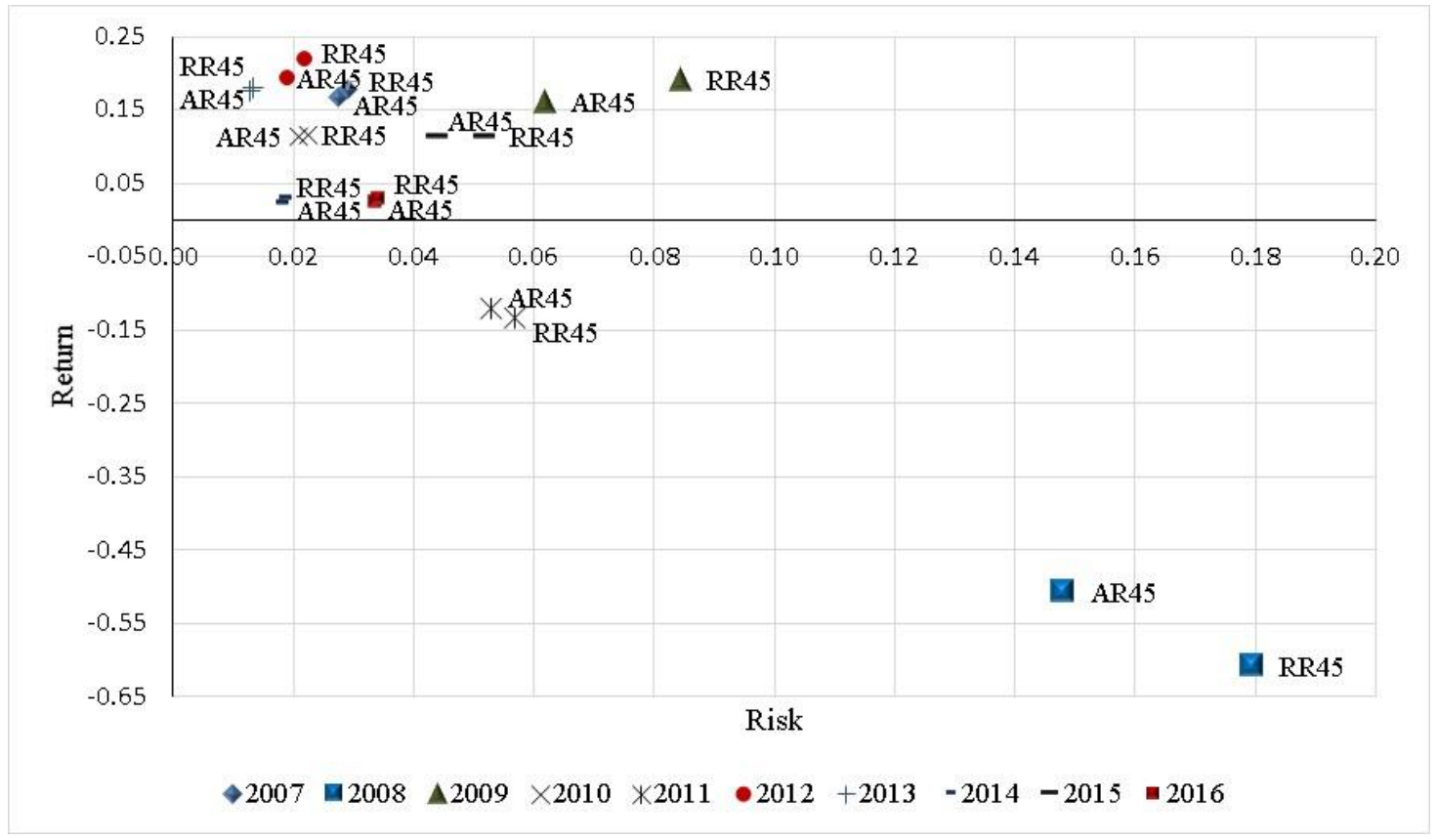

Figure 7: Out-of-sample risks and returns of RR and AR portfolios computed by out-of-sample year and considering an in-sample period length of 4 years and $a$ and $a$ value of 5 for the risk aversion parameter.

First, the AR portfolio is a dominant solution comparatively to the RR portfolio only in the outof-sample years where all the optimal portfolios, presented in the empirical application, revealed poor performances, namely in the out-of-sample years 2008 and 2011, previously described as periods of high volatility and, simultaneously, low mean returns. Second, and for the remaining out-of-sample years, the RR portfolio generally presents identical risks levels (except in 2009 and 2015) while it always presents higher returns, comparatively to the AR portfolio. 
Out-of-sample regret and $S_{I}$ support the previous outcome. In fact, the RR solution outperforms the AR portfolio when the $S_{I}$ measure is considered (see Table 4 and Table 6) while it is systematically more robust, revealing lower regrets than the AR portfolio, in most of the windows (see Table 5 and Table 6).

Table 6: Out-of-sample regret and Sharpe ratio $\left(S_{I}\right)$ of the AR portfolio (by out-of-sample year and considering an in-sample period length of 4 years and a value of 5 for the risk aversion parameter)

\begin{tabular}{ccccccccccc}
\hline Measure & 2007 & 2008 & 2009 & 2010 & 2011 & 2012 & 2013 & 2014 & 2015 & 2016 \\
\hline $\boldsymbol{S}_{\boldsymbol{I}}$ & 0.782 & -0.210 & 0.579 & 0.732 & -0.029 & 1.402 & 1.564 & 0.177 & 0.544 & 0.160 \\
Regret & 0.113 & 26.045 & 0.127 & 0.157 & 0.517 & 0.075 & 0.111 & 0.208 & 0.159 & 0.253 \\
\hline
\end{tabular}

Although results are not presented for the rest of the parameter combinations, the previous findings generally prevail regardless of the in-sample period length and the value of the risk aversion parameter. Moreover, it was also observed that, although reducing the in-sample period length has an improving effect in the overall performance of both the AR and RR portfolios, this effect is more substantial in the RR portfolio. Finally, an important fact concerning the investor's risk preferences was also observed. Since the absolute robust approach is described in the robust optimization theory as a more conservative methodology, it would be expected a better performance of the AR portfolio for higher levels of the risk aversion parameter. While, on the one hand the AR portfolio always presents lower (mean) risk comparatively to the RR portfolio ( 0.058 versus 0.065 , when $\gamma=0.5$; and 0.044 versus 0.051 , when $\gamma=5$ ), on the other hand, it reveals worst (mean) risk-adjusted return, measured by the $S_{\mathrm{I}}(0.528$ versus 0.529 when $\gamma=0.5$, and 0.570 versus 0.578 when $\gamma=5$ ). The values previously presented were verified for an insample period of 4 years. These results clearly reinforce the relevance of the relative robust methodology within the robust portfolio theory.

\section{Conclusions and future research}

In this paper, a new methodology for computing relative-robust portfolios based on the optimization of the investor's expected utility is presented. In this approach, regret is defined as the utility loss for the investor resulting from choosing a given portfolio instead of choosing the optimal portfolio of the realized scenario for the uncertain parameters. This approach was compared to the absolute robust approach, which seeks to maximize the minimum investor's expected utility. These approaches were implemented assuming that the investor's preferences are described by a power utility function.

To avoid reaching a solution strongly dependent on the underlying in-sample data, several estimation subsamples were gathered from the in-sample data and for each subsample a minimax regret and a maximin solutions are computed. The robust portfolios are defined as the ones presenting the best performance in the worst-case, and are then tested in the out-of-sample set and compared with non-robust and robust portfolios described in the literature. A genetic algorithm is applied for the computation of the proposed robust portfolios; for the minimax regret solutions, it allowed the transformation of a 3-level optimization problem in a 2-level problem. Results are analyzed assuming different in-sample period lengths and values of the risk aversion parameter, and relevant conclusions are drawn regarding the real benefits of the proposed methodology from the investors' perspective.

The results suggest that reducing the in-sample period length for the estimation of the model parameters increases the exposure of the portfolios to individual stocks, since the diversification level of the computed portfolios decreases. Regardless of the in-sample period length, it can be observed that the RR portfolio is highly diversified, assigning non-zero weights to the majority of the assets.

Analyzing the effect of the in-sample period length in the portfolios' out-of-sample performance, it is quite evident that reducing the in-sample period length seems to generally improve the overall performance of both the AR and RR portfolios; also, this effect is more substantial in the RR portfolio. Concerning the out-of-sample performance of the non-robust portfolios, reducing the 
in-sample period length seems to improve the overall performance of the GMV while substantially deteriorating the out-of-sample performance of the MV portfolio.

The overall results regarding the performance of the computed portfolios support previous findings concerning the sensitivity of the MV portfolio to estimation error and the effects of the input uncertainty in the optimization process (Best and Grauer, 1991b; Chopra and Ziemba, 1993; Jagannathan and Ma, 2003; DeMiguel, Garlappi and Uppal, 2009), as well as the good performance of the GMV portfolio (Chan, Karceski and Lakonishok, 1999; Jagannathan and Ma, 2003).

Regarding the proposed relative robust methodology, it generates optimal portfolios that consistently present low risk, and (non-negative) attractive returns. In fact, the RR portfolio outperforms the MV portfolio and the EW portfolio, in many of the windows under analysis, which enhances the relevance of the proposed methodology among non-robust portfolio optimization methodologies. When compared to the minimax regret model presented by (Xidonas et al., 2017), the proposed relative robust approach seems to provide more consistent results concerning the out-of-sample performance of the generated portfolios. Standing out as one of the very few optimal portfolios with no poor performances, the robustness and the consistency between in-sample and out-of-sample performance of the proposed relative-robust portfolio was confirmed, concerning utility loss for the investor (regret) and, although not so outstanding, concerning the proximity to its expected performance. Furthermore, the underperformance of the RR portfolio occurs in time windows characterized by out-of-sample periods of high volatility and, simultaneously, low assets mean returns, where the majority of the optimal portfolios perform poorly.

The comparison of the out-of-sample performances of the RR and AR portfolios allowed to draw important conclusions concerning the real benefits of the proposed methodology from the investors' perspective. The findings suggest that the proposed RR portfolio generally outperforms the proposed AR portfolio, even when higher levels of risk aversion are considered. In this sense, the authors consider that the considered relative robust and absolute robust methodologies contribute to reinforce the relevance of robust optimization methodologies, in particular of relative robust models, within the field of portfolio selection under uncertainty.

Future research will include the application of the proposed methodology to different datasets as well as the analysis of the performance of the AR and RR portfolios for other levels of the relative risk aversion parameter.

Since the out-of-sample performance of relative-robust portfolios has been little explored, further empirical applications are needed in order to find the real benefits for the investors of using robust methodologies, particularly the relative robust approach, when making investment decisions.

\section{Acknowledgements}

This study has been funded by national funds, through FCT, Portuguese Science Foundation, under project UID/Multi/00308/2019.

\section{References}

Artzner, P. et al. (1999) 'Coherent measures of risk', Mathematical finance. Wiley Online Library, 9(3), pp. 203-228.

de Athayde, G. M. and Flôres, R. G. (2004) 'Finding a maximum skewness portfolio-a general solution to three-moments portfolio choice', Journal of Economic Dynamics and Control, 28(7), pp. 1335-1352. doi: https://doi.org/10.1016/S0165-1889(02)00084-2.

Ben-Tal, A., Bertsimas, D. and Brown, D. B. (2010) 'A soft robust model for optimization under ambiguity', Operations research. INFORMS, 58(4-part-2), pp. 1220-1234.

Ben-Tal, A., El Ghaoui, L. and Nemirovski, A. (2009) Robust optimization. Princeton: Princeton University Press.

Ben-Tal, A. and Nemirovski, A. (1998) 'Robust Convex Optimization', Mathematics of Operations 
Research. INFORMS, 23(4), pp. 769-805. doi: 10.1287/moor.23.4.769.

Bernoulli, D. (1954) 'Exposition of a new theory on the measurement of risk', Econometrica: Journal of the Econometric Society. JSTOR, pp. 23-36.

Bertsimas, D. and Thiele, A. (2006) 'Robust and data-driven optimization: Modern decision-making under uncertainty', INFORMS tutorials in operations research: models, methods, and applications for innovative decision making, p. 137.

Best, M. J. and Grauer, R. R. (1991a) 'On the sensitivity of mean-variance-efficient portfolios to changes in asset means: some analytical and computational results.', Review of Financial Studies. Oxford University Press / USA, 4(2), p. 315.

Best, M. J. and Grauer, R. R. (1991b) 'Sensitivity analysis for mean-variance portfolio problems', Management Science. INFORMS: Institute for Operations Research, 37(8), pp. 980-989.

Biagini, S. and Pınar, M. Ç. (2017) 'The robust Merton problem of an ambiguity averse investor', Mathematics and Financial Economics, 11(1), pp. 1-24. doi: 10.1007/s11579-016-0168-6.

Bondt, W. F. M. and Thaler, R. (1985) 'Does the stock market overreact?', The Journal of finance. Wiley Online Library, 40(3), pp. 793-805.

Chan, L. K. C., Karceski, J. and Lakonishok, J. (1999) 'On portfolio optimization: forecasting covariances and choosing the risk model.', Review of Financial Studies. Oxford University Press / USA, 12(5).

Chopra, V. K. and Ziemba, W. T. (1993) 'The effect of errors in means, variances, and covariances on optimal portfolio choice', Journal of Portfolio Management. World Scientific, 19(2), pp. 6-11.

Clarke, R., De Silva, H. and Thorley, S. (2002) 'Portfolio constraints and the fundamental law of active management', Financial Analysts Journal. CFA Institute, 58(5), pp. 48-66.

Constantinides, G. M. and Malliaris, A. G. (1995) 'Chapter 1 Portfolio theory', in R.A. Jarrow, V. M. and W. T. Z. B. T.-H. in O. R. and M. S. (ed.) Finance. Elsevier, pp. 1-30. doi: http://dx.doi.org/10.1016/S09270507(05)80045-3.

Cornuejols, G. and Tütüncü, R. (2006) Optimization Methods in Finance. Cambridge: Cambridge University Press (Mathematics, Finance and Risk).

Cremers, J.-H., Kritzman, M. and Page, S. (2003) 'Portfolio formation with higher moments and plausible utility', Revere Street Working Paper Series 272-12.

Cremers, J.-H., Kritzman, M. and Page, S. (2005) 'Optimal hedge fund allocations', The Journal of Portfolio Management. Institutional Investor Journals Umbrella, 31(3), pp. 70-81.

Deb, K. (2011) 'Multi-objective optimisation using evolutionary algorithms: an introduction', in Multiobjective evolutionary optimisation for product design and manufacturing. Springer, pp. 3-34.

DeMiguel, V., Garlappi, L. and Uppal, R. (2009) 'Optimal Versus Naive Diversification: How Inefficient is the 1/N Portfolio Strategy?', Review of Financial Studies. Oxford University Press / USA, 22(5), pp. 1915-1953.

Doerner, K. et al. (2004) 'Pareto ant colony optimization: A metaheuristic approach to multiobjective portfolio selection', Annals of Operations Research, 131(1-4), pp. 79-99. doi: 10.1023/B:ANOR.0000039513.99038.c6.

Elton, E. J. and Gruber, M. J. (1992) 'Portfolio analysis with a nonnormal multi-index return-generating process', Review of Quantitative Finance and Accounting. Springer, 2(1), pp. 5-16.

Fabozzi, F. J. et al. (2007) Robust portfolio optimization and management. John Wiley \& Sons.

Fabretti, A., Herzel, S. and Pınar, M. Ç. (2014) 'Delegated portfolio management under ambiguity aversion', Operations Research Letters, 42(2), pp. 190-195. doi: https://doi.org/10.1016/j.orl.2014.02.002. Fama, E. F. (1996) 'Multifactor portfolio efficiency and multifactor asset pricing', Journal of financial and quantitative analysis. Cambridge University Press, 31(4), pp. 441-465.

Fama, E. F. and French, K. R. (1992) 'The cross-section of expected stock returns', the Journal of Finance. Wiley Online Library, 47(2), pp. 427-465.

Fisher, I. (1906) 'The nature of capital and income'. New York: Macmillan.

Francis, J. C. and Kim, D. (2013) Modern Portfolio Theory,+ Website: Foundations, Analysis, and New Developments. John Wiley \& Sons.

Gabrel, V., Murat, C. and Thiele, A. (2014) 'Recent advances in robust optimization: An overview', European Journal of Operational Research, 235(3), pp. 471-483. doi: http://dx.doi.org/10.1016/j.ejor.2013.09.036.

Gen, M. and Cheng, R. (2000) Genetic algorithms and engineering optimization. John Wiley \& Sons.

El Ghaoui, L. and Lebret, H. (1997) 'Robust Solutions to Least-Squares Problems with Uncertain Data', SIAM Journal on Matrix Analysis and Applications. Society for Industrial and Applied Mathematics, 18(4), pp. 1035-1064. doi: 10.1137/S0895479896298130.

Goldfarb, D. and Iyengar, G. (2003) 'Robust portfolio selection problems', Mathematics of Operations Research. INFORMS, 28(1), pp. 1-38.

Gregory, C., Darby-Dowman, K. and Mitra, G. (2011) 'Robust optimization and portfolio selection: The 
cost of robustness', European Journal of Operational Research, 212(2), pp. 417-428. doi: http://dx.doi.org/10.1016/j.ejor.2011.02.015.

Halldórsson, B. V and Tütüncü, R. H. (2003) 'An Interior-Point Method for a Class of Saddle-Point Problems', Journal of Optimization Theory and Applications. Kluwer Academic Publishers-Plenum Publishers, 116(3), pp. 559-590. doi: 10.1023/A:1023065319772.

Harvey, C. R. et al. (2010) 'Portfolio selection with higher moments', Quantitative Finance. Routledge, 10(5), pp. 469-485. doi: 10.1080/14697681003756877.

Hauser, R., Krishnamurthy, V. and Tütüncü, R. (2013) 'Relative robust portfolio optimization', arXiv preprint arXiv: 1305.0144.

Hendricks, D. (1997) 'Evaluation of value-at-risk models using historical data', Economic Policy Review. CFA Institute, 2(1).

Ingersoll, J. E. (1987) Theory of financial decision making. Rowman \& Littlefield.

Israelsen, C. L. (2005) 'A refinement to the Sharpe ratio and information ratio.', Journal of Asset Management. Palgrave Macmillan Ltd., 5(6), pp. 423-427.

Jagannathan, R. and Ma, T. (2003) 'Risk Reduction in Large Portfolios: Why Imposing the Wrong Constraints Helps', The Journal of Finance. Blackwell Publishing, 58(4), pp. 1651-1684. doi: 10.1111/1540-6261.00580.

Jean, W. H. (1971) 'The Extension of Portfolio Analysis to Three or More Parameters', The Journal of Financial and Quantitative Analysis. Cambridge University Press, 6(1), pp. 505-515. doi: $10.2307 / 2330125$.

Jegadeesh, N. and Titman, S. (1993) 'Returns to buying winners and selling losers: Implications for stock market efficiency', The Journal of finance. Wiley Online Library, 48(1), pp. 65-91.

Jin, Y. and Branke, J. (2005) 'Evolutionary optimization in uncertain environments-a survey', IEEE Transactions on evolutionary computation. IEEE, 9(3), pp. 303-317.

Kalä1, R., Lamboray, C. and Vanderpooten, D. (2012) 'Lexicographic $\alpha$-robustness: An alternative to minmax criteria', European Journal of Operational Research, 220(3), pp. 722-728. doi: http://dx.doi.org/10.1016/j.ejor.2012.01.056.

Kim, J. H., Kim, W. C. and Fabozzi, F. J. (2013) 'Composition of robust equity portfolios', Finance Research Letters, 10(2), pp. 72-81. doi: http://dx.doi.org/10.1016/j.frl.2013.02.001.

Kim, W. C. et al. (2013) 'What do robust equity portfolio models really do?', Annals of Operations Research. Springer US, 205(1), pp. 141-168. doi: 10.1007/s10479-012-1247-6.

Kim, W. C., Fabozzi, F. J., et al. (2014) 'Controlling portfolio skewness and kurtosis without directly optimizing third and fourth moments', Economics Letters, 122(2), pp. 154-158. doi: http://dx.doi.org/10.1016/j.econlet.2013.11.024.

Kim, W. C., Kim, M. J., et al. (2014) 'Robust portfolios that do not tilt factor exposure', European Journal of Operational Research, 234(2), pp. 411-421. doi: http://dx.doi.org/10.1016/j.ejor.2013.03.029.

Kim, W. C., Kim, J. H. and Fabozzi, F. J. (2014) 'Deciphering robust portfolios', Journal of Banking \& Finance, 45, pp. 1-8. doi: http://dx.doi.org/10.1016/j.jbankfin.2014.04.021.

Kouvelis, P. and Yu, G. (1997) 'Robust Discrete Optimization: Past Successes and Future Challenges', in Robust Discrete Optimization and Its Applications. Boston, MA: Springer US, pp. 333-356. doi: 10.1007/978-1-4757-2620-6_9.

Levy, H. and Markowitz, H. M. (1979) 'Approximating expected utility by a function of mean and variance', The American Economic Review. JSTOR, 69(3), pp. 308-317.

Lu, Z. (2006) 'A new cone programming approach for robust portfolio selection', Optimization Methods \& Software, 26(1), pp. 89-104.

Lu, Z. (2011) 'Robust portfolio selection based on a joint ellipsoidal uncertainty set', Optimization Methods \& Software. Taylor \& Francis, 26(1), pp. 89-104.

Markowitz, H. (1952) 'Portfolio Selection', The Journal of Finance. Blackwell Publishing Ltd, 7(1), pp. 77-91. doi: 10.1111/j.1540-6261.1952.tb01525.x.

Markowitz, H. (1959) Portfolio Selection: Efficient Diversification of Investments. New York: John Wiley \& Sons.

Marschak, J. (1938) 'Money and the Theory of Assets', Econometrica, Journal of the Econometric Society. JSTOR, pp. 311-325.

Natarajan, K., Pachamanova, D. and Sim, M. (2009) 'Constructing risk measures from uncertainty sets', Operations Research. INFORMS, 57(5), pp. 1129-1141.

Von Neumann, J. and Morgenstern, O. (1947) Theory of games and economic behavior. Princeton: Princeton University Press.

Paç, A. B. and Pınar, M. Ç. (2014) 'Robust portfolio choice with CVaR and VaR under distribution and mean return ambiguity', Top. Springer, 22(3), pp. 875-891.

Paç, A. B. and Pınar, M. Ç. (2018) 'On robust portfolio and naïve diversification: mixing ambiguous and 
unambiguous assets', Annals of Operations Research, 266(1), pp. 223-253. doi: 10.1007/s10479-0172619-8.

Pınar, M. Ç. (2016) 'On robust mean-variance portfolios’, Optimization. Taylor \& Francis, 65(5), pp. 1039_ 1048.

Pınar, M. Ç. and Burak Paç, A. (2014) 'Mean semi-deviation from a target and robust portfolio choice under distribution and mean return ambiguity', Journal of Computational and Applied Mathematics, 259, pp. 394-405. doi: https://doi.org/10.1016/j.cam.2013.06.028.

Rockafellar, R. T. and Uryasev, S. (2000) 'Optimization of conditional value-at-risk', Journal of risk, 2, pp. 21-42.

Roll, R. and Ross, S. (1994) 'On the Cross-sectional Relation between Expected Returns and Betas', The Journal of Finance. Wiley/Blackwell (10.1111), 49(1), pp. 101-121. doi: 10.1111/j.15406261.1994.tb04422.x.

Ross, S. A. (1976) 'The arbitrage theory of capital asset pricing', Journal of economic theory. Elsevier, 13(3), pp. 341-360.

Roy, B. (2010) 'Robustness in operational research and decision aiding: A multi-faceted issue', European Journal of Operational Research. Elsevier, 200(3), pp. 629-638.

Scherer, B. and Xu, X. (2007) 'The Impact of Constraints on Value-Added', The Journal of Portfolio Management, 33(4), p. 45 LP-54.

Sharpe, W. F. (1964) 'Capital asset prices: A theory of market equilibrium under conditions of risk', The journal of finance. Wiley Online Library, 19(3), pp. 425-442.

Shoaf, J. and Foster, J. A. (1998) 'The efficient set GA for stock portfolios', in 1998 IEEE International Conference on Evolutionary Computation Proceedings. IEEE World Congress on Computational Intelligence (Cat. No.98TH8360), pp. 354-359. doi: 10.1109/ICEC.1998.699758.

Steuer, R. E., Qi, Y. and Hirschberger, M. (2008) 'Portfolio selection in the presence of multiple criteria', in Handbook of financial engineering. Springer, pp. 3-24.

Szegö, G. (2005) 'Measures of risk', European Journal of Operational Research, 163(1), pp. 5-19. doi: http://dx.doi.org/10.1016/j.ejor.2003.12.016.

Xidonas, P. et al. (2017) 'Robust multiobjective portfolio optimization: A minimax regret approach', European Journal of Operational Research, 262(1), pp. 299-305. doi: https://doi.org/10.1016/j.ejor.2017.03.041.

Yaman, H., Karasan, O. E. and Pinar, M. C. (2007) 'Restricted robust optimization for maximization over uniform matroid with interval data uncertainty', Mathematical Programming, 110(2), pp. 431-441.

Yang, X. (2006) 'Improving portfolio efficiency: A genetic algorithm approach', Computational Economics. Springer, 28(1), p. 1.

Zopounidis, C. and Doumpos, M. (2002) 'Multi-criteria decision aid in financial decision making: methodologies and literature review', Journal of Multi-Criteria Decision Analysis. John Wiley \& Sons, Ltd., 11(4-5), pp. 167-186. doi: 10.1002/mcda.333. 